\title{
Rotation curves and metallicity gradients from HII regions in spiral galaxies $\star, \star \star$
}

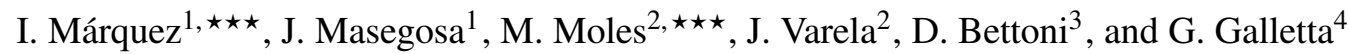 \\ 1 Instituto de Astrofísica de Andalucía (CSIC), Apartado 3004, 18080 Granada, Spain \\ 2 Instituto de Matemáticas y Física Fundamental (CSIC), C) Serrano 113B, 28006 Madrid, Spain \\ 3 Osservatorio Astronomico di Padova, Vicolo Osservatorio 5, 35122 Padova, Italy \\ ${ }^{4}$ Dipartimento di Astronomia, Università di Padova, Vicolo Osservatorio 2, 35122 Padova, Italy
}

Received 24 December 2001/ Accepted 25 June 2002

\begin{abstract}
In this paper we study long slit spectra in the region of $\mathrm{H} \alpha$ emission line of a sample of 111 spiral galaxies with recognizable and well defined spiral morphology and with a well determined environmental status, ranging from isolation to non-disruptive interaction with satellites or companions. The form and properties of the rotation curves are considered as a function of the isolation degree, morphological type and luminosity. The line ratios are used to estimate the metallicity of all the detected HII regions, thus producing a composite metallicity profile for different types of spirals. We have found that isolated galaxies tend to be of later types and lower luminosity than the interacting galaxies. The outer parts of the rotation curves of isolated galaxies tend to be flatter than in interacting galaxies, but they show similar relations between global parameters. The scatter of the Tully-Fisher relation defined by isolated galaxies is significantly lower than that of interacting galaxies. The $[\mathrm{NII}] / \mathrm{H} \alpha$ ratios, used as a metallicity indicator, show a clear trend between $Z$ and morphological type, $t$, with earlier spirals showing higher ratios; this trend is tighter when instead of t the gradient of the inner rotation curve, $G$, is used; no trend is found with the change in interaction status. The $Z$-gradient of the disks depends on the type, being almost flat for early spirals, and increasing for later types. The $[\mathrm{NII}] / \mathrm{H} \alpha$ ratios measured for disk HII regions of interacting galaxies are higher than for normal/isolated objects, even if all the galaxy families present similar distributions of $\mathrm{H} \alpha$ Equivalent Width.
\end{abstract}

Key words. galaxies: spiral - galaxies: kinematics and dynamics - galaxies: structure - galaxies: interactions

\section{Introduction}

The analysis of the rotation curves of disk galaxies is the most direct way to obtain information on the mass distribution of spiral galaxies. The ionized gas has been used for a long time as a tracer of their kinematics. During the 80's, Rubin and collaborators started a systematic effort to obtain accurate rotation curves of spiral galaxies of all morphological types and luminosity (Rubin et al. 1991, and references therein). The accumulation of data from different sources helped to get an overall

\footnotetext{
Send offprint requests to: I. Márquez, e-mail: isabel@iaa.es

* Tables 3 and 4 and Figs. 6, 7 and 21 are only available in electronic form at http://www.edpsciences.org. Table 5 is only available in electronic form at the CDS via anonymous ftp to cdsarc.u-strasbg.fr (130.79.128.5) or via

http://cdsweb.u-strasbg.fr/cgi-bin/qcat?J/A+A/393/389

$\star \star$ Based on data obtained Asiago/Ekar Observatory. Also based on observations made with INT operated on the island of La Palma by ING in the Spanish Observatorio del Roque de Los Muchachos of the Instituto de Astrofísica de Canarias.

$\star \star \star$ Visiting Astronomer, German-Spanish Astronomical Center, Calar Alto, operated by the Max-Planck-Institut fur Astronomie jointly with the Spanish National Commission for Astronomy.
}

picture of the form of the rotation curve of spirals, and its relation to other galactic properties. It is now recognized that the maximum rotational velocity, $V_{\mathrm{m}}$, is related to the total mass (and luminosity) of the galaxy, the optical scale radius of the disk and the morphological type (see Persic et al. 1996). The flatness of the outer rotation curve in most cases also led to acceptance of the existence of massive dark halos in spiral galaxies (see Rubin et al. 1991; Sofue 1998).

Most of those analyses were based on data sets assembled with no completeness criteria. In particular, the galaxies were considered as field or cluster objects, and no further attention was payed to their environmental status, in spite of the expected influence of even small companions on the mass distribution and star formation history of a given galaxy. Spiral galaxies in very close isolated pairs were studied by Keel (1993, 1996). Trying to understand the effects of the interaction on the dynamics of disk galaxies, Márquez \& Moles (1996; hereafter Paper I) studied the properties of isolated (see below for the definition of isolation) spiral galaxies, to set a zero-point for the effects of the interaction; see also Mathewson et al. 1992, and Courteau 1997, for an analysis of field spirals). Márquez \& Moles (1999; hereafter Paper II), also studied the 
properties of spirals in isolated pairs, and compared them to those of the isolated galaxies in Paper I. It was found that the main differences is the presence of type II disk profiles in interacting systems (but not in isolated galaxies), and a flatter outer rotation curve in isolated galaxies. No distorted curves were found among isolated disk galaxies.

More recently, 2D Fabry-Perot rotation curves have been obtained for a number of cluster spirals in order to determine the environmental effects in such large aggregates. The results show a complex pattern (Amram et al. 1996). Barton et al. (2000, 2001) have also analyzed the rotation curves of spiral galaxies in close pairs and in the general field in order to put some constraints on the cluster effects on the kinematical properties of galaxies, and the consequences in their use for distance estimation by means of the Tully-Fisher relation. Their results do confirm the earlier results in Paper II, in the sense of a more scattered T-F relation for non-isolated objects. Similarly, galaxies with interacting companions in the recent analysis by Kannappan et al. (2002) fall on the high luminosity/low velocity width side of the TF and show more scatter.

We emphasize that the so-called field galaxies should be carefully investigated since some of them could still be perturbed by small companions or satellites, that could produce significant effects (Athanassoula 1984; Conselice \& Gallagher 1999; Conselice et al. 2000). In Papers I and II a quantitative criterion of isolation was given, trying to identify truly isolated objects to build up a reference for the analysis of the effects of gravitational interaction. We will use a similar approach here.

The same long slit spectroscopic data used for the study of the gas kinematics can also be used, through the flux ratios of the observed emission lines, to trace the metallicity, $Z$, along the disk, and to determine the existence of $Z$-gradients. The existing analysis points out that the global metallicity is related to the mass (hence, to $V_{\mathrm{m}}$ ), and that $Z$ decreases gently outwards (see the review by Henry \& Worthey 1999). Ferguson et al. (1998) have extended the analysis towards the extreme outer regions of disks, finding that $Z$ drops there abruptly, but keeping values far from pristine.

In the present paper, we will study a sample of 111 spiral galaxies with a well studied environmental status, ranging from isolation to mild interaction with satellites or companions. In all cases however the interaction is non disruptive (they have been selected to have recognizable and well defined spiral morphology). The data comprise new long slit spectroscopy for 85 spiral galaxies. The remaining data are from Paper I. The form and properties of the rotation curves are considered as a function of the isolation degree, morphological type and luminosity. The line ratios are used to estimate the metallicity of all the detected HII regions, thus producing a composite metallicity profile for different types of spirals.

Section 2 is devoted to the description of the sample and the determination of the interaction status. In Sect. 3, the observations and data reduction procedures are presented. Sections 4 and 5 deal with the rotation curves and the Tully-Fisher relation, respectively. In Sect. 6 the properties of nuclear and extranuclear HII regions are described. The summary and conclusions are given in Sect. 7.

\section{The sample}

The 85 galaxies with new long slit data come from three sources:

- Automatically selected isolated galaxies from the CfA catalogue (Huchra et al. 1999), among those brighter than $m_{B}=13$ (and not bigger than $4^{\prime}$ in diameter, for practical reasons), with an inclination between $32^{\circ}$ and $73^{\circ}$, to minimize the uncertainties with the correction in both photometric and kinematic parameters. The isolation criterium for automatic selection was that they had no CfA companions (i.e., with known red-shift) within a projected distance of $0.5 \mathrm{Mpc}$, or with a red-shift difference greater than $500 \mathrm{~km} \mathrm{~s}^{-1}$ (see Márquez 1994; Paper I). A total of 43 galaxies were selected in that way.

- An additional sample of 29 spiral galaxies also from the CfA catalogue, also imposing the automatical isolation criteria and with $|b| \geq 40^{\circ}$ but with no restrictions in apparent magnitude, size or inclination.

- Thirteen spiral galaxies in 7 pairs taken from the catalogue of isolated pairs by Karachentsev (1972). We applied the automatic isolation criterium to the pair as a whole. In addition to this, only those galaxies with still recognizable and well defined morphology were retained. Therefore, we implicitly avoid strongly interacting galaxies, with about $40 \%$ of pairs being widely separated, in contrast to previous studies of pairs of spirals, where mostly close pairs were selected (Keel 1993, 1996; Barton et al. 2001).

We have also included in our final sample a total of 26 spiral galaxies (17 isolated and 9 spirals in $5 \mathrm{~S}$-S pairs) from Paper $\mathrm{I}^{1}$, so the analysis refers to the whole sample of 111 spiral galaxies. Notice that since we imposed that the candidates would have a well defined spiral morphology, and no bright (CfA) companions, our sample does not include cases of very strong and/or disruptive interaction. Nevertheless, even in those mildly interacting systems, the perturbations can be recognizable depending, other than on the properties of the galaxy itself, on the mass, size, distance and relative velocity of the perturbing agent.

\subsection{The isolation status revisited}

As a second step for defining a sample of isolated galaxies, the previously selected as isolated were investigated for the presence of perturbing companions. It is well known that the influence of small, close companions can produce secular alterations on the dynamics of the primary system (Athanassoula 1984; Sundelius et al. 1987; Byrd \& Howard 1992). Moreover, the effects can manifest themselves on very different time scales. To cope with all those aspects, we define a galaxy as isolated when the possible past perturbations by neighboring

\footnotetext{
1 Isolated galaxies: NGC 818, UGC 3511, UGC 3804, NGC 2532, NGC 2712, IC 529, NGC 3294, NGC 3549, NGC 3811, NGC 3835, NGC 4162, NGC 4290, NGC 4525, NGC 5962, NGC 6155, NGC 6395, NGC 6643; spirals in Karachentsev's pairs: NGC 2798/99, NGC 4567/68, NGC 3646, NGC 7469/IC 5283, NGC 7537/41.
} 
objects, if any, have been completely erased by now. Accepting that typical time scales for the decay of the perturbation effects are not longer than a few times $10^{9}$ years, a criterion as given before can be defined.

Given the incompleteness of the CfA catalog, and the lack of red-shift information for many of the possible companions, we have adopted the parameter $f$, defined as $f=3 \times \log (r / D)+$ $0.4 \times\left(m-m_{\mathrm{p}}\right)^{2}$ to describe the environmental status of our sample galaxies. Numerical results (Athanassoula 1984) indicate that detectable effects are expected for $f$ larger than -2 . To take into account the possible past effects, we have adopted as truly isolated those galaxies with $f \leq-4$ (the details and justification of the adopted criterion will be given in Varela et al., in preparation). The search for companions was done with a catalog complete to $m_{B}=18$, kindly made available to us by G. Paturel. The results were visually inspected to discard any candidate that is not a galaxy. As a result, we have an indication of the status of each galaxy just given by the maximum of the $f$ values corresponding to all its (candidate) companions.

According to that status we have classified our sample galaxies into 3 groups. The first one includes 24 truly isolated galaxies in the sense defined before (interaction class $I N T=1)$; the second, with 44 objects, includes possibly interacting galaxies, with a companion (with $\log f>-4$ ) of unknown red-shift $(I N T=2)$; it will most probably contain both truly isolated and truly interacting galaxies and will therefore be considered separately from the others. Group 3, with 43 objects, is for interacting galaxies, including the 22 galaxies in Karachentsev's pairs and the 21 isolated spirals from the authomatic rearch that resulted to have confirmed companion(s). The type and absolute magnitude distributions of the two subgroups included in the last Interaction Grop are similar. We have performed the Kolmogorov-Smirnov test and found that they are not significantly different, and therefore can be considered as defining the same group.

\subsection{Characterization of the sample}

We have considered the morphology and luminosity of the whole sample of galaxies to see if the selection criteria would have resulted in differences among the galaxies with different status. The type and magnitude are from the RC3 catalogue. The distribution of the types is shown in Fig. 1. It appears that 37 galaxies have $t<4$, and 47 have $4 \leq t<6$ ). The remaining 27 galaxies with $t \geq 6(24 \%)$ (12 galaxies with $t=6)$ present different morphological peculiarities, but in all cases an underlying disk does exist.

We have compared the morphology of the confirmed isolated galaxies (Interacting Type 1, 24 objects) with that of interacting galaxies (Interacting Type 3, 43 objects). As can be seen in Fig. 1, both $t$-distributions look different, in particular due to the lack of galaxies with $t<3$, plus the scarcity of $t=3$ galaxies (only 4) among the isolated objects in our sample. To quantify the differences we performed a

\footnotetext{
${ }^{2} D$ is the distance from the target galaxy to the perturber, $r$ is the radius of the target galaxy, $m$ is the magnitude of the target galaxy and $m_{\mathrm{p}}$ is the magnitude of the perturber.
}
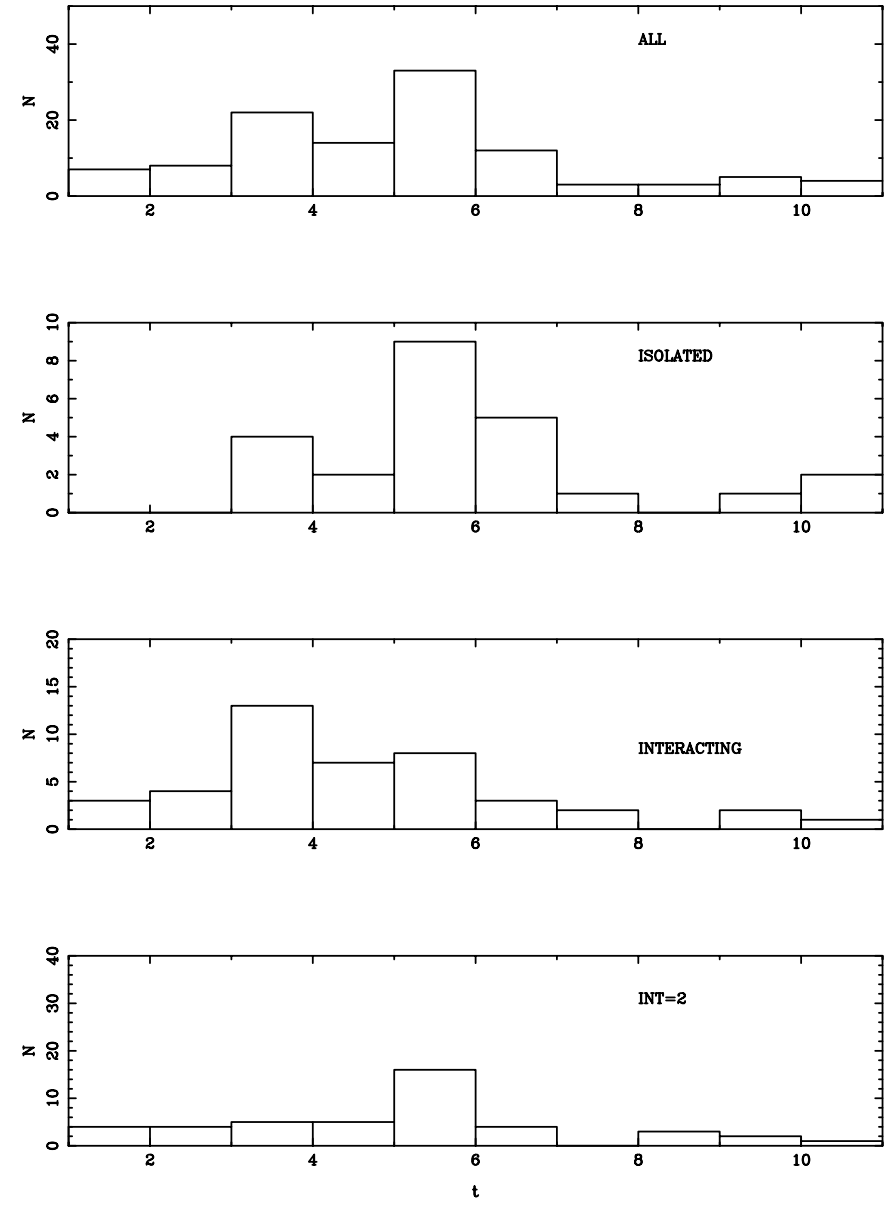

Fig. 1. Distribution of morphological types in our sample.

Kolmogorov-Smirnov test, finding that, at the $95 \%$ level, the difference is statistically significant. The same kind of result is found when only galaxies with $t<7$ are considered.

Regarding the presence of bars for the whole sample, 32 are barred systems (SB code in the RC3), 24 are of intermediate type (SX), and 34 have been classified as unbarred systems (SA). We notice that these numbers are similar to those reported in studies on the fraction of barred spirals based on optical studies (see for instance Moles et al. 1995; Ho et al. 1997). For the other 21 galaxies there is no information about the presence of a bar in the RC3. The same percentage of isolated objects $(33 \%)$ is found among barred and unbarred galaxies.

The distribution of absolute magnitudes for the whole sample is given in Fig. 2. The absolute magnitude ranges from -16.31 to -22.43 , the low luminosity tail corresponding to late type galaxies with morphological classification later than $t=6$. For those late types alone, the median value is -18.63 , whereas it reaches -20.42 for the other types. Looking at those galaxies with $t \leq 6$, we find that the interacting objects tend to be brighter, $M_{B}=-20.69 \mathrm{mag}$, versus $M_{B}=-20.28 \mathrm{mag}$ for isolated objects, with a dispersion of 0.62 mag in both cases.

Both the morphological type and absolute magnitude distributions for the whole sample are similar to those of the spiral galaxies analyzed by de Jong \& van der Kruit (1994), who also selected non-disrupted spiral galaxies. We have also compared with the sample by Jansen et al. (2000): considering galaxies 

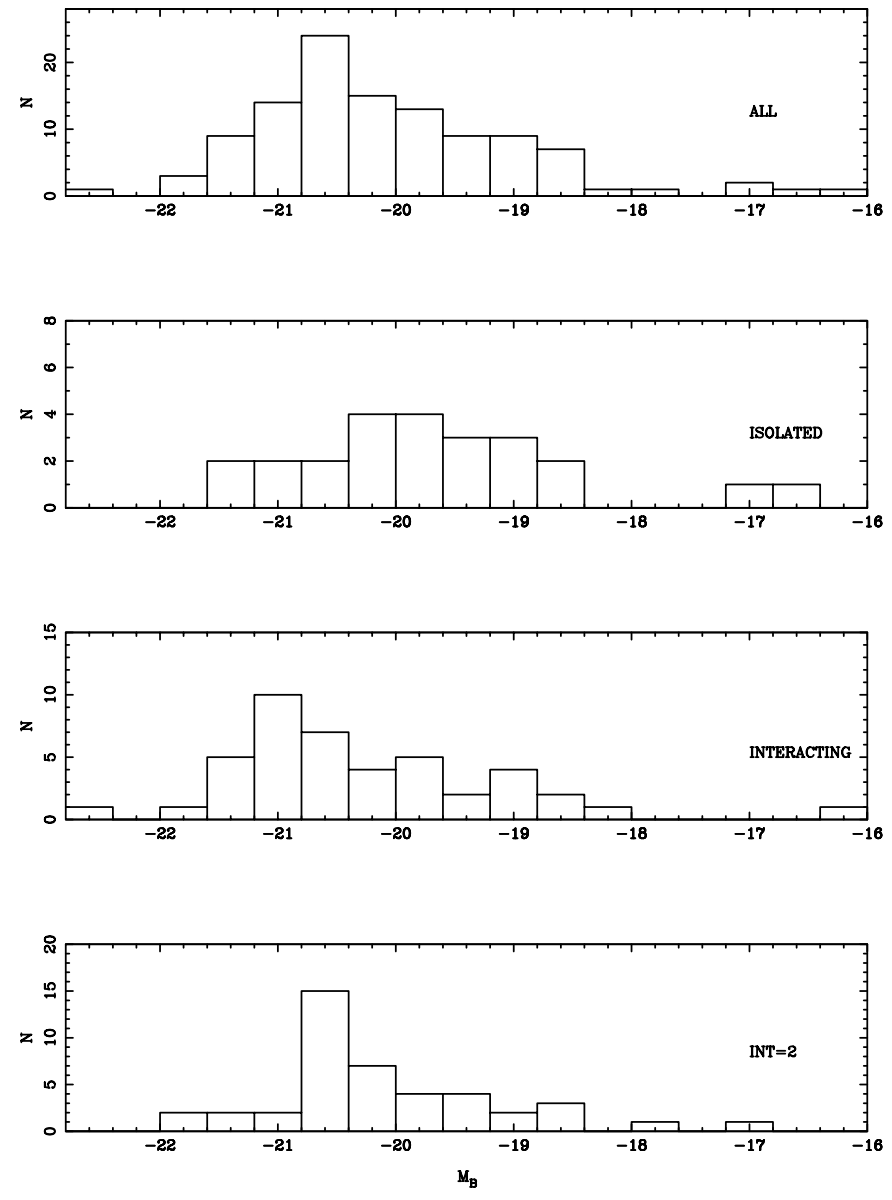

Fig. 2. Distribution of $B$ absolute magnitudes.

defined as isolated with our first criteria, i.e., with no companions within a projected distance of $0.5 \mathrm{Mpc}$, or with a red-shift difference greater than $500 \mathrm{~km} \mathrm{~s}^{-1}$, the $t$-distribution is similar to that of our galaxies, except that their selection method produces more $t \geq 7$ galaxies and (by construction) a much flatter distribution of absolute magnitudes (see Fig. 3). Indeed, the Kolmogorov-Smirnov test for galaxies earlier than $t=7$ (92 and 79 galaxies in Jansen et al. sample and in our sample, respectively) gives the result that both distributions are not statistically different. We also note that only one galaxy among the 8 secondary members of the isolated pairs has $t=3$, the rest been later types. Therefore, we are confident that our sample galaxies is representative of normal spirals and it is not biased to any particular property, and the differences between isolated and interacting galaxies seem to be real.

\section{Observations and data reductions}

The spectroscopic data were collected with three different instruments: the Cassegrain Spectrograph attached to the $2.2 \mathrm{~m}$ telescope in Calar Alto (CAHA, Spain), the Intermediate Dispersion Spectrograph at the $2.5 \mathrm{~m}$ Isaac Newton telescope in La Palma (Spain), and the Boller \& Chivens spectrograph attached to the $1.82 \mathrm{~m}$ Asiago-Ekar telescope at the Asiago Observatory (Italy). The setup and main characteristics of the observations are given in Table 1.
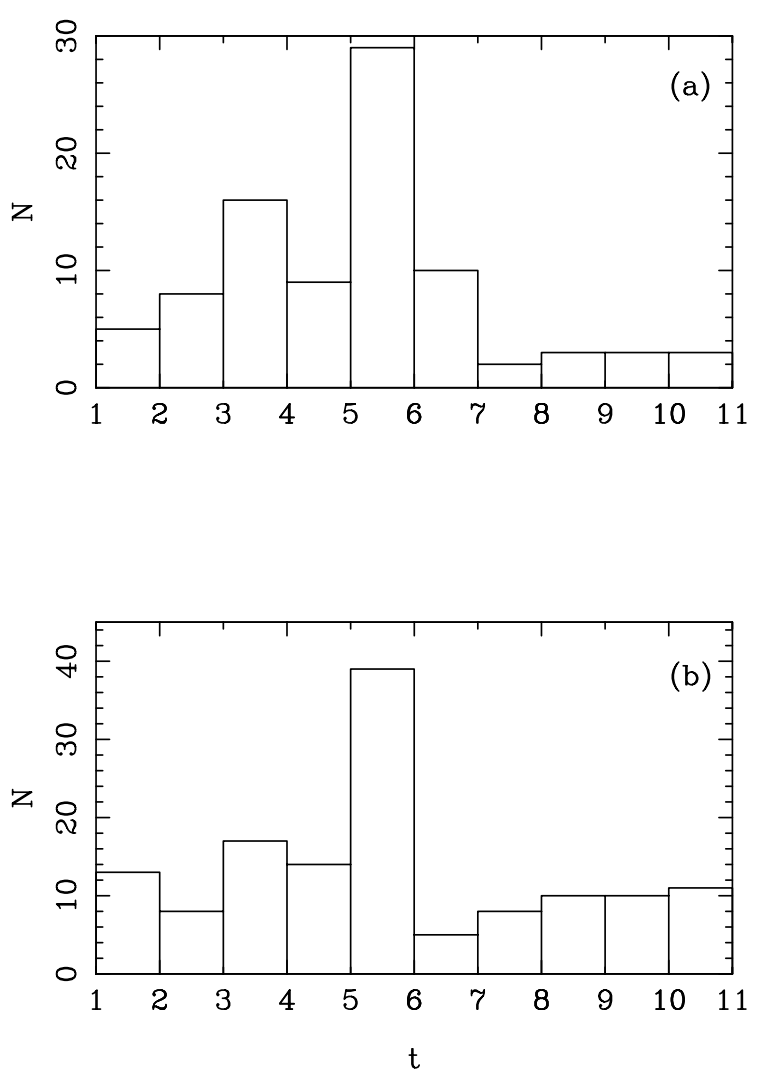

Fig. 3. Comparison of the distribution of morphological types for authomatically selected isolated galaxies in our sample a) and in Jansen's sample b).

All the galaxies were observed with the slit oriented along the galaxy major axis. We determined the PA of the axis from our own broad band images (Paper I), or from DSS images. In most cases the values we measured are very close to that catalogued in the RC3. In general the differences are within $15^{\circ}$. Only in three cases did we find a big discrepancy, namely for N3769a $\left(40^{\circ}\right), \mathrm{N} 3044\left(100^{\circ}\right)$, and U11577 $\left(85^{\circ}\right)$. In those cases we adopted our own values for the PA of the major axis. The PA are given in Table 2, together with the exposure time. For 22 galaxies we also obtained spectra along one additional $\mathrm{PA}$, corresponding in 16 cases to the minor axis.

The data obtained in Calar Alto and La Palma were reduced in a similar way, using the appropriate routines in FIGARO. After bias subtraction, and flat field correction, the data were wavelength calibrated using He-Ar comparison spectra, that were observed before and after each object exposure. The resulting accuracy was tested using the sky lines [OI] $\lambda 6300$, and [OI] 66364 , as references. The centroids of the lines were measured section by section. The average value was then compared with the reference wavelengths to fix the zero-point offset of each spectrum, and the rms error as an indication of the error in the resulting velocity distribution induced by the residual distortion (see Table 2).

The sky background level was determined taking median averages over two strips at both sides of the galaxy signal. The parameters of the lines were measured with the program LINES kindly provided to us by Dr. Perea. This program perform a 
Table 1. Observations.

\begin{tabular}{lccccccc}
\hline \hline Telescope & Date & Instrument & $\begin{array}{c}\text { Spectral res. } \\
(\AA / \mathrm{A} / \mathrm{x})\end{array}$ & $\begin{array}{c}\text { Range } \\
(\AA)\end{array}$ & $\begin{array}{c}\text { Slit width } \\
(\operatorname{arcsec})\end{array}$ & $\begin{array}{c}\text { Spatial res. } \\
(\operatorname{arcsec} / \mathrm{pix})\end{array}$ & $\begin{array}{c}\text { Average seeing } \\
(\operatorname{arcsec})\end{array}$ \\
\hline 2.2 m CAHA & Jun. 1991 & Boller \& Chivens & 1.31 & $5926-7151$ & 1.5 & 1.35 & $1.2-1.5$ \\
$2.2 \mathrm{~m}$ CAHA & Mar. 1993 & Boller \& Chivens & 1.36 & $5736-7138$ & 1.5 & 1.69 & $1.1-1.4$ \\
$I N T$ & Feb. 1993 & IDS & 0.79 & $6000-7000$ & 1.5 & 0.65 & 1.1 \\
ASIAGO & 1996,1997 & Boller \& Chivens & 0.96 & $5000-7000$ & 2.5 & 1.16 & $1.4-2.4$ \\
\hline
\end{tabular}

simultaneous interactive polynomial fitting of the continuum and Gaussian fitting of the emission selected lines providing the Equivalent Width, central wavelength of the Gaussian fit and the integrated fluxes of the lines. The errors in Table 5 have been calculated by quadratic addition of photon counting errors and the errors in the continuum level determination.

We used cross-correlation techniques as described in Paper I to extract the kinematic information. The spatial section in the 2D spectrum with the highest $S / N$ ratio was used as a template for the cross-correlation. The errors refer to the determination of the velocity shift with respect to the template spectrum. They are shown as the error bars in all the velocity distributions (see Figs. 4 and 5).

The data obtained at Asiago (a total of 29 galaxies) was reduced using IRAF routines, with the same criteria and definitions as for the other data.

\section{The shape of the rotation curves}

The long slit spectra along the major axis we obtained contain adequate information to elaborate the rotation curves for 78 out of the 85 galaxies. They are presented in Figs. 4 and 5. The 7 galaxies with velocity distributions not reliable enough to trace their rotation curves are NGC 3507, NGC 5394, NGC 5641, NGC 2344, NGC 1036, NGC 5375 and UGC 11577. The parameters derived from our rotation curves (without further correction) are given in Tables 3 and 4 .

The kinematic center was defined as that section for which the differences between the two branches of the rotation curve (in particular, for the most symmetric region which normally corresponds to the central parts), are minimized. In most cases, within our resolution, that center corresponds to the photometric center, i.e., the absolute maximum in the continuum distribution along the slit. The red-shift corresponding to that kinematical center was adopted as the red-shift of the system in all cases and is given in Tables 3 and 4 . The derived distances were determined allowing for correction for galactocentric motion, following the $\mathrm{RC} 2$, and for virgocentric motion. Since the galaxies are rather nearby, the last correction should be considered. It was calculated following Kraan-Korteweg (1986; model 1). We found that those corrections typically amount to less than $15 \%$. The distances calculated from the corrected values and $H_{0}=75 \mathrm{~km} \mathrm{~s}^{-1} \mathrm{Mpc}^{-1}$ are given in Tables 3 and 4 .

We have also obtained 21 additional spectra; 16 of them along the minor axis. The resulting velocity distributions are shown in Figs. 6 and 7. We notice that for NGC 2543 and NGC 5963 the velocity distributions along the quoted minor axes show velocity amplitudes for the outskirts of about $50 \mathrm{~km} \mathrm{~s}^{-1}$, implying that a misalignment exists between photometric and kinematical minor axes. For NGC 7217 and NGC 7479 the resulting amplitude is much higher, up to about $100 \mathrm{~km} \mathrm{~s}^{-1}$. The presence of non-circular motions is clearly detected in the central regions of NGC 1530, NGC 2543, NGC 5351, NGC 5480, NGC 5656, NGC 5963, NGC 7177, NGC 7217 and NGC 7479. We note that, excepting NGC 5656 and NGC 5963, for which the detailed morphological classification is missing in the RC3 catalogue, the other galaxies are classified as having rings, and four of them are weakly (NGC 7177) or strongly (NGC 1530, NGC 2543, NGC 7479) barred. Non-circular motions are detectable in 3 out of the 5 non-major-axis spectra, namely NGC 5394, NGC 5899 and NGC 2906, in the inner 5, 10 and 7 arcsec, respectively. The exact connection between morphological and kinematical features, for which the imaging is needed, is out the scope of this paper. We just note that the percentage of galaxies showing non-circular motions $(11 \%)$ is in agreement with the $17 \%$ reported by Rubin et al. (1997).

Different parameters are used to describe the rotation curves depending on their final purpose. We have measured all the parameters that could be of some interest for statistical purposes. In particular we have measured the first local maximum, $V_{\text {max }}$, in the observed velocity distribution, and the corresponding distance to the center, $R_{\max }$. We have also measured the slope, $\mathrm{G}$ (in $\mathrm{km} \mathrm{s}^{-1} / \mathrm{kpc}$ ) of the inner rotation curve. This parameter, directly related to the bulge-to-disk ratio, appears as one of the two main parameters (together with the total mass or luminosity) to describe the family of isolated spiral galaxies and correlates with the bulge-to-disk ratio better than the morphological type (Papers I and II and see below). Indeed, we also measured the absolute maximum velocity, $V_{\mathrm{m}}$, and the central distance at which it is reached, $R_{\mathrm{m}}$, and the maximum extent of the observed rotation curve, $R_{\mathrm{M}}$. All those parameters show relations between them and with other galactic properties. Thus, as shown in Fig. 8, later type galaxies tend to have lower $G$ values (see also Paper II and Baiesi-Pillastrini 1987). A better correlation was found between the same slope $G$, and a quantitative descriptor of the morphological type as the bulge to disk ratio, $B / D$, in particular for isolated galaxies (Paper II). Unfortunately, the available photometric data in the literature is not abundant and homogeneous enough to further test that relation here. 

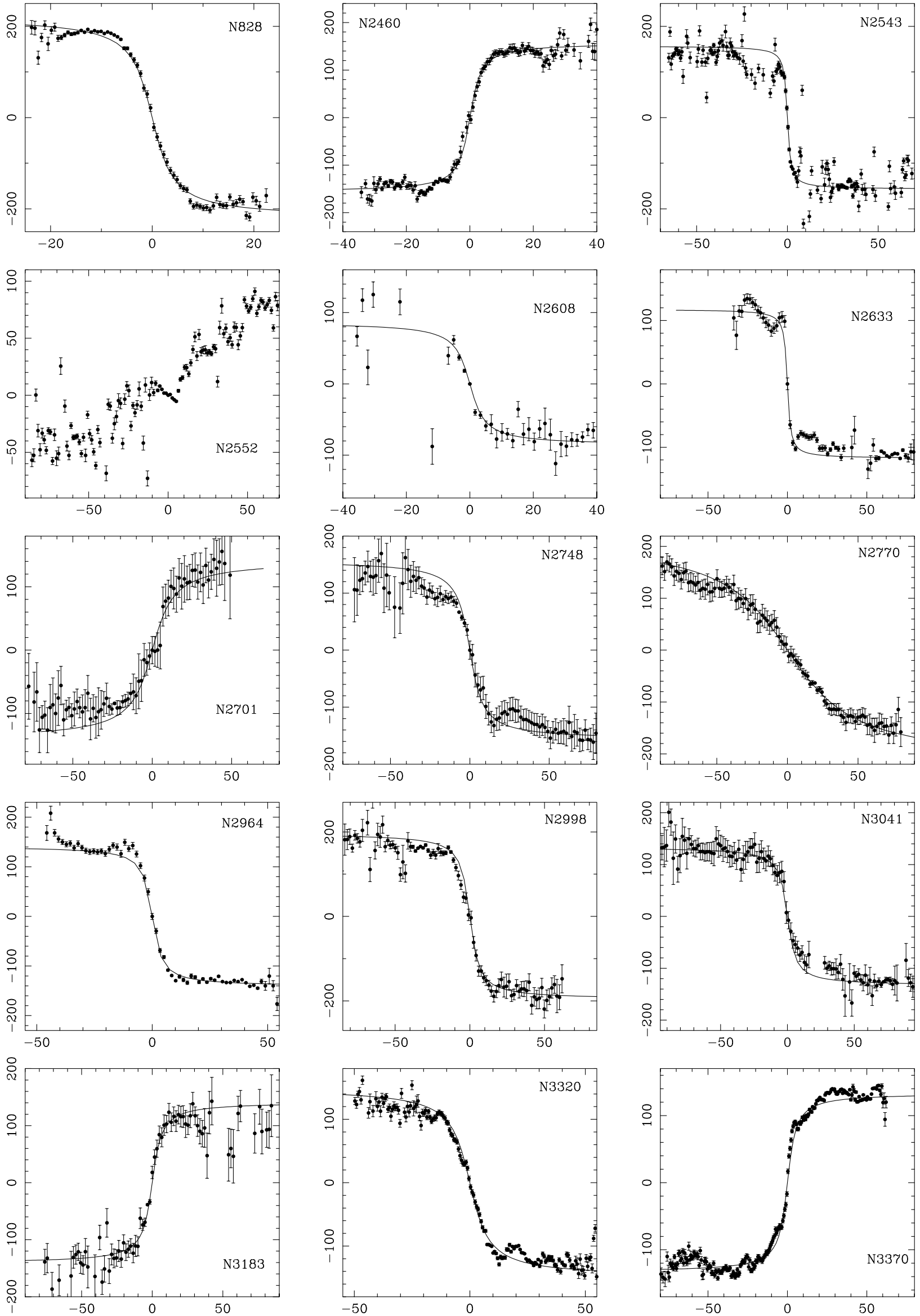

Fig. 4. Rotation curves from INT and CAHA spectra. Velocities are in $\mathrm{km} \mathrm{s}^{-1}$ ( $y$ axis) and distances to the center in arcseconds. 
I. Márquez et al.: Rotation curves and metallicities of spiral galaxies


Fig. 4. continued. 

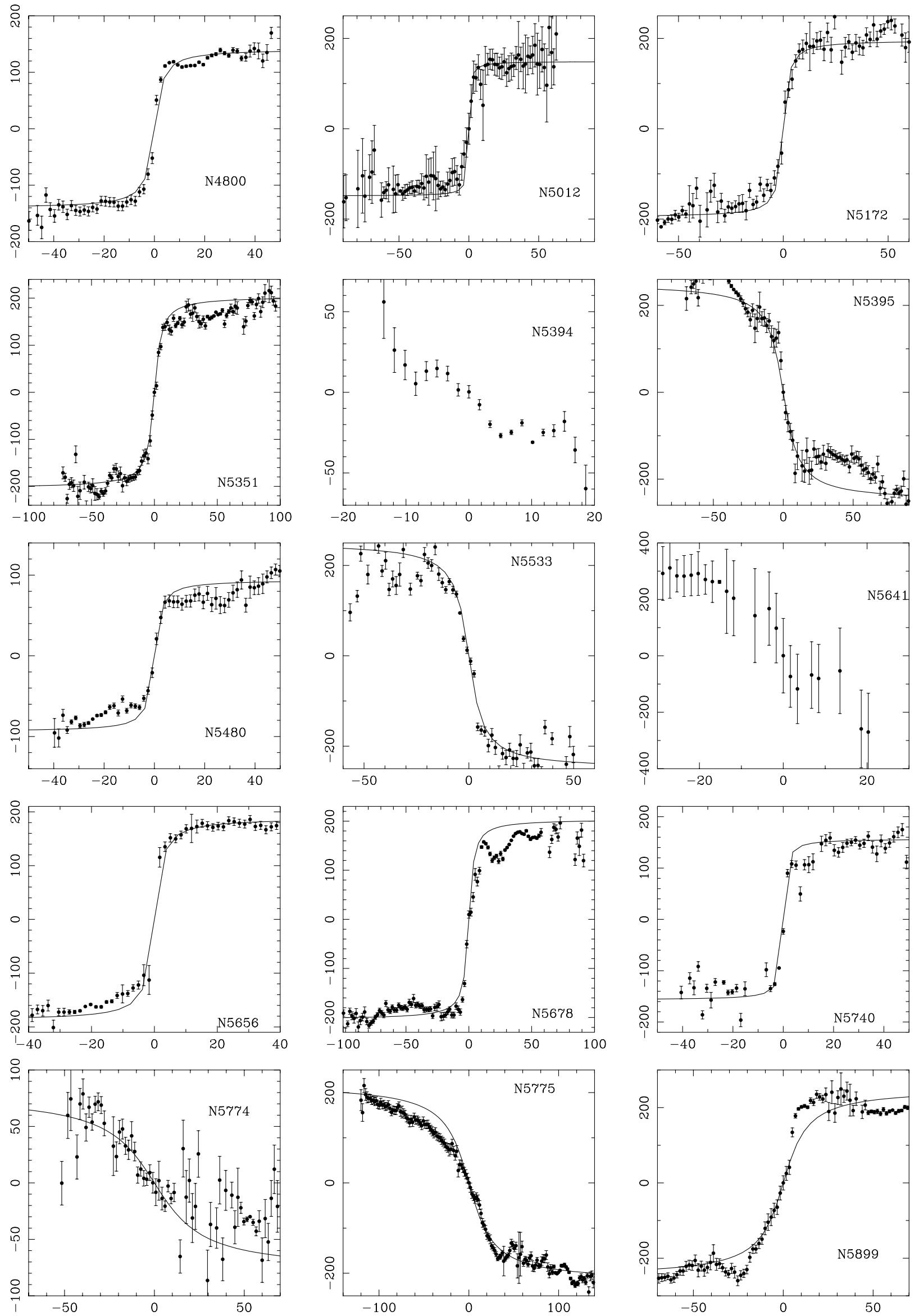

Fig. 4. continued. 



Fig. 4. continued.

Regarding the outer part of the rotation curve, a descriptor was defined (Papers I and II) for its behavior,

$\Delta=\arctan \left[\left(\left(v_{\mathrm{m}}-v_{\max }\right) / \max \left(v_{\mathrm{m}}, v_{\max }\right)\right) \times\left(r_{\mathrm{m}}-r_{\max }\right) / r_{25}\right]$.

It takes values around zero for flat rotation curves, whereas it is positive for rising curves and negative for declining curves. The distribution of this parameter for isolated and interacting is shown in Fig. 9. We find that for isolated galaxies $\Delta=0 \pm 7$, and for interacting galaxies $\Delta=5 \pm 16$. This result confirms and extends the earlier one reported in Paper II, where galaxies in isolated pairs were compared to isolated galaxies.

Sometimes a different parameter is adopted to describe the gradient of the outer rotation curve, defined as (see for instance Dale et al. 2001),

$O G=\left[V\left(0.70 \times R_{25}\right)-V\left(0.35 \times R_{25}\right)\right] / V\left(0.70 \times R_{25}\right)$. 

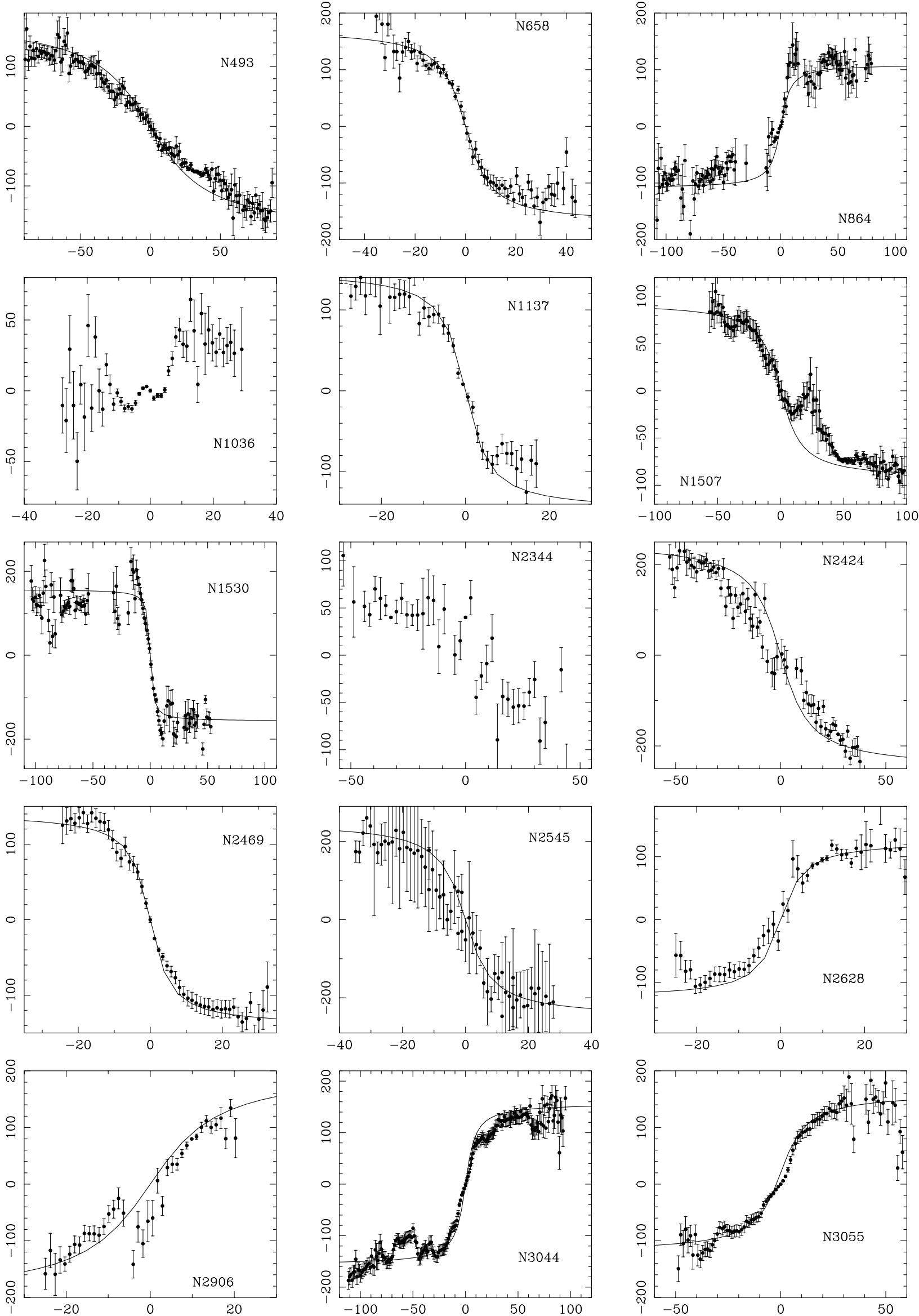

Fig. 5. Rotation curves from Asiago spectra. 

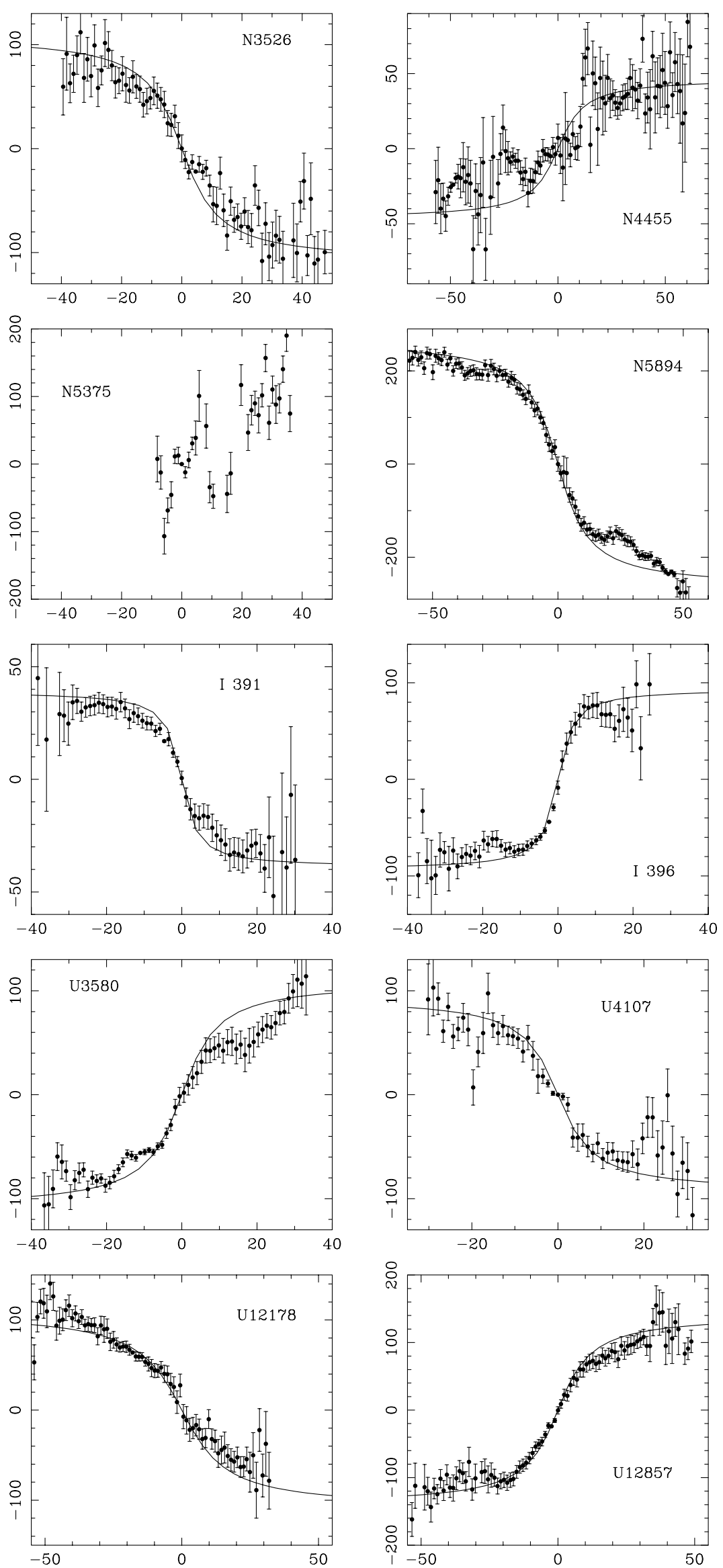

Fig. 5. continued.
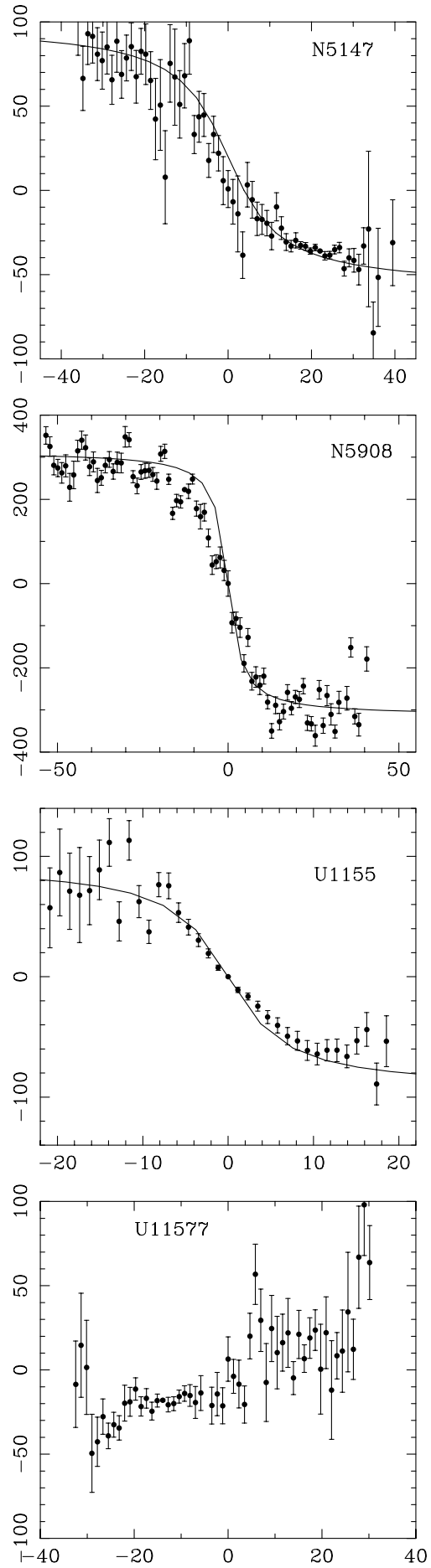
Table 2. Detailed log of the spectra.

\begin{tabular}{|c|c|c|c|c|c|c|c|c|c|}
\hline Galaxy & Date & $\mathrm{PA}$ & Time & RMS & Galaxy & Date & $\mathrm{PA}$ & Time & RMS \\
\hline NGC 828 & $02 / 16 / 93$ & 100 & 3600 & 1.4 & NGC 493 & $29 / 10 / 97$ & 58 & 3600 & 1.5 \\
\hline NGC 2460 & $02 / 16 / 93$ & 40 & 3600 & 2.0 & NGC 658 & $28 / 10 / 97$ & 20 & 2983 & 2.1 \\
\hline \multirow[t]{2}{*}{ NGC 2543} & $02 / 12 / 93$ & 45 & 3600 & 4.9 & NGC 864 & $05 / 10 / 96$ & 20 & 3600 & 1.9 \\
\hline & $02 / 17 / 93$ & 145 & 3600 & 3.1 & NGC 1036 & $30 / 12 / 97$ & 5 & 1803 & 3.0 \\
\hline NGC 2552 & $02 / 17 / 93$ & 45 & 3600 & 1.4 & NGC 1137 & $05 / 10 / 96$ & 20 & 1463 & 2.8 \\
\hline NGC 2608 & $03 / 27 / 93$ & 60 & 3000 & 1.0 & NGC 1507 & $28 / 12 / 97$ & 11 & 3600 & 1.6 \\
\hline NGC 2633 & $03 / 26 / 93$ & 175 & 3000 & 0.6 & NGC 1530 & $27 / 12 / 97$ & 23 & 3600 & 0.9 \\
\hline NGC 2701 & $03 / 28 / 93$ & 23 & 3600 & 1.3 & & $27 / 12 / 97$ & 113 & 3600 & 1.3 \\
\hline NGC 2748 & $03 / 29 / 93$ & 38 & 3000 & 1.9 & NGC 2344 & $28 / 12 / 97$ & 0 & 3600 & 2.5 \\
\hline NGC 2770 & $03 / 26 / 93$ & 148 & 3000 & 0.5 & & $28 / 12 / 97$ & 90 & 3600 & 3.0 \\
\hline NGC 2964 & $03 / 30 / 93$ & 97 & 3000 & 0.5 & NGC 2424 & $29 / 01 / 96$ & 81 & 3600 & 2.5 \\
\hline NGC 2998 & $03 / 29 / 93$ & 53 & 3000 & 0.4 & NGC 2469 & $29 / 01 / 96$ & 40 & 3600 & 3.4 \\
\hline NGC 3041 & $03 / 28 / 93$ & 95 & 3600 & 0.4 & NGC 2545 & 05/06/97 & 170 & 3600 & 1.8 \\
\hline NGC 3183 & $03 / 29 / 93$ & 170 & 3000 & 0.3 & NGC 2628 & $31 / 10 / 97$ & 177 & 3600 & 4.0 \\
\hline NGC 3320 & $02 / 12 / 93$ & 20 & 3600 & 3.1 & & $31 / 10 / 97$ & 87 & 2000 & 2.9 \\
\hline NGC 3370 & $02 / 14 / 93$ & 148 & 3600 & 2.5 & NGC 2906 & $11 / 04 / 97$ & 75 & 3600 & 2.5 \\
\hline NGC 3395 & $03 / 29 / 93$ & 50 & 1800 & 0.7 & & $11 / 04 / 97$ & 120 & 3600 & 3.0 \\
\hline NGC 3396 & $03 / 28 / 93$ & 100 & 3600 & 0.5 & NGC 3044 & 04/03/97 & 114 & 3600 & 3.1 \\
\hline NGC 3471 & $02 / 13 / 93$ & 14 & 3600 & 4.4 & NGC 3055 & $12 / 04 / 97$ & 63 & 3428 & 2.5 \\
\hline NGC 3501 & $03 / 30 / 93$ & 25 & 3000 & 1.0 & & $12 / 04 / 97$ & 83 & 3600 & 1.9 \\
\hline NGC 3507 & $03 / 27 / 93$ & 110 & 3000 & 0.4 & NGC 3526 & $05 / 03 / 97$ & 55 & 3600 & 0.9 \\
\hline NGC 3689 & $02 / 13 / 93$ & 97 & 3600 & 3.0 & NGC 4455 & $05 / 03 / 97$ & 16 & 3600 & 1.6 \\
\hline NGC 3769a & $03 / 31 / 93$ & 110 & 3000 & 0.3 & NGC 5147 & $14 / 04 / 96$ & 0 & 3600 & 2.6 \\
\hline NGC 3769 & $03 / 31 / 93$ & 152 & 3000 & 1.0 & NGC 5375 & $12 / 04 / 97$ & 0 & 3600 & 0.6 \\
\hline NGC 3976 & $03 / 27 / 93$ & 53 & 3000 & 1.0 & NGC 5894 & $12 / 04 / 97$ & 13 & 3600 & 3.0 \\
\hline NGC 4047 & $02 / 16 / 93$ & 105 & 3600 & 3.5 & NGC 5908 & $05 / 03 / 97$ & 154 & 2339 & 2.1 \\
\hline NGC 4284 & $03 / 29 / 93$ & 102 & 3600 & 0.5 & IC 391 & $28 / 12 / 97$ & 0 & 3600 & 0.8 \\
\hline NGC 4389 & $03 / 28 / 93$ & 105 & 3000 & 0.5 & IC 396 & $29 / 12 / 97$ & 85 & 3600 & 1.5 \\
\hline NGC 4496a & $02 / 17 / 93$ & 70 & 3200 & 10 & & $29 / 12 / 97$ & 175 & 3600 & 2.0 \\
\hline NGC $4496 \mathrm{~b}$ & $03 / 27 / 93$ & 115 & 3000 & 1.0 & UGC 1155 & $28 / 10 / 97$ & 165 & 3600 & 0.8 \\
\hline NGC 4793 & $02 / 13 / 93$ & 50 & 3600 & 1.7 & UGC 3580 & $29 / 12 / 97$ & 3 & 3600 & 3.0 \\
\hline NGC 4800 & $03 / 27 / 93$ & 25 & 3000 & 0.5 & UGC 4107 & $29 / 10 / 97$ & 40 & 3600 & 2.6 \\
\hline \multirow[t]{2}{*}{ NGC 5012} & $06 / 22 / 91$ & 10 & 1800 & 4.3 & & $29 / 10 / 97$ & 130 & 3600 & 1.9 \\
\hline & $06 / 17 / 91$ & 170 & 1800 & 6.5 & UGC 11577 & $28 / 10 / 97$ & 90 & 3600 & 3.0 \\
\hline \multirow[t]{2}{*}{ NGC 5172} & $06 / 19 / 91$ & 103 & 2500 & 3.3 & UGC 12178 & $29 / 10 / 97$ & 20 & 3600 & 1.9 \\
\hline & 06/19/91 & 13 & 1000 & 3.1 & UGC 12857 & $05 / 10 / 96$ & 34 & 3600 & 2.4 \\
\hline \multirow[t]{2}{*}{ NGC 5351} & $06 / 20 / 91$ & 150 & 2000 & 4.2 & & & & & \\
\hline & $06 / 22 / 91$ & 10 & 1000 & 6.0 & & & & & \\
\hline \multirow{2}{*}{ NGC 5394} & $06 / 18 / 91$ & 145 & 1000 & 19 & & & & & \\
\hline & $06 / 23 / 91$ & 55 & 1200 & 20 & & & & & \\
\hline NGC 5395 & 06/19/91 & 170 & 2000 & 3.9 & & & & & \\
\hline \multirow[t]{2}{*}{ NGC 5480} & $06 / 22 / 91$ & 177 & 1500 & 5.5 & & & & & \\
\hline & $06 / 22 / 91$ & 87 & 1000 & 6.5 & & & & & \\
\hline NGC 5533 & $06 / 21 / 91$ & 30 & 1800 & 4.5 & & & & & \\
\hline NGC 5641 & 06/19/91 & 158 & 2000 & 4.3 & & & & & \\
\hline \multirow[t]{2}{*}{ NGC 5656} & $06 / 21 / 91$ & 50 & 2000 & 3.3 & & & & & \\
\hline & $06 / 22 / 91$ & 140 & 1000 & 4.9 & & & & & \\
\hline NGC 5678 & $06 / 21 / 91$ & 5 & 1800 & 2.9 & & & & & \\
\hline NGC 5740 & $06 / 21 / 91$ & 160 & 2000 & 2.2 & & & & & \\
\hline NGC 5774 & $06 / 20 / 91$ & 142 & 2000 & 4.5 & & & & & \\
\hline NGC 5775 & $06 / 20 / 91$ & 146 & 1800 & 4.2 & & & & & \\
\hline \multirow[t]{2}{*}{ NGC 5899} & $06 / 18 / 91$ & 18 & 2000 & 10 & & & & & \\
\hline & $06 / 17 / 91$ & 162 & 2500 & 5.1 & & & & & \\
\hline NGC 5963 & $06 / 19 / 91$ & 45 & 2000 & 4.9 & & & & & \\
\hline & $06 / 22 / 91$ & 135 & 1200 & 7.1 & & & & & \\
\hline NGC 5970 & $06 / 21 / 91$ & 88 & 1800 & 5.9 & & & & & \\
\hline & $06 / 23 / 91$ & 178 & 953 & 7.5 & & & & & \\
\hline NGC 6070 & $06 / 17 / 91$ & 62 & 2500 & 9.3 & & & & & \\
\hline & $06 / 23 / 91$ & 152 & 1200 & 1.6 & & & & & \\
\hline NGC 6106 & $06 / 20 / 91$ & 140 & 1800 & 6.7 & & & & & \\
\hline & $06 / 22 / 91$ & 50 & 1000 & 4.5 & & & & & \\
\hline NGC 6181 & $06 / 20 / 91$ & 175 & 2000 & 5.8 & & & & & \\
\hline NGC 6207 & $06 / 21 / 91$ & 22 & 1402 & 9.0 & & & & & \\
\hline NGC 6239 & $06 / 18 / 91$ & 118 & 2000 & 6.0 & & & & & \\
\hline NGC 7177 & 06/18/91 & 90 & 2000 & 4.5 & & & & & \\
\hline & $06 / 23 / 91$ & 0 & 1200 & 6.7 & & & & & \\
\hline NGC 7217 & $06 / 24 / 91$ & 100 & 1200 & 3.7 & & & & & \\
\hline & $06 / 22 / 91$ & 10 & 1200 & 3.9 & & & & & \\
\hline NGC 7448 & $06 / 21 / 91$ & 170 & 1800 & 5.5 & & & & & \\
\hline NGC 7479 & $06 / 24 / 91$ & 45 & 1200 & 5.2 & & & & & \\
\hline & $06 / 24 / 91$ & 135 & 1200 & 6.4 & & & & & \\
\hline
\end{tabular}

February 1993: INT telescope; 1996, 1997: Asiago, otherwise: 2.2 m CAHA telescope.

We found $O G=7.8 \pm 6.6$ for isolated galaxies and $10.4 \pm 15.5 \quad$ Regarding the maximum velocity ${ }^{3}, V_{\mathrm{m}}$, it has been for interacting galaxies, showing that both parameters, $\Delta$ and known that it is also related to the morphological type $O G$, give similar information about the outer rotation curve.

${ }^{3}$ All the velocities have been corrected for inclination following Peterson et al. (1978). 


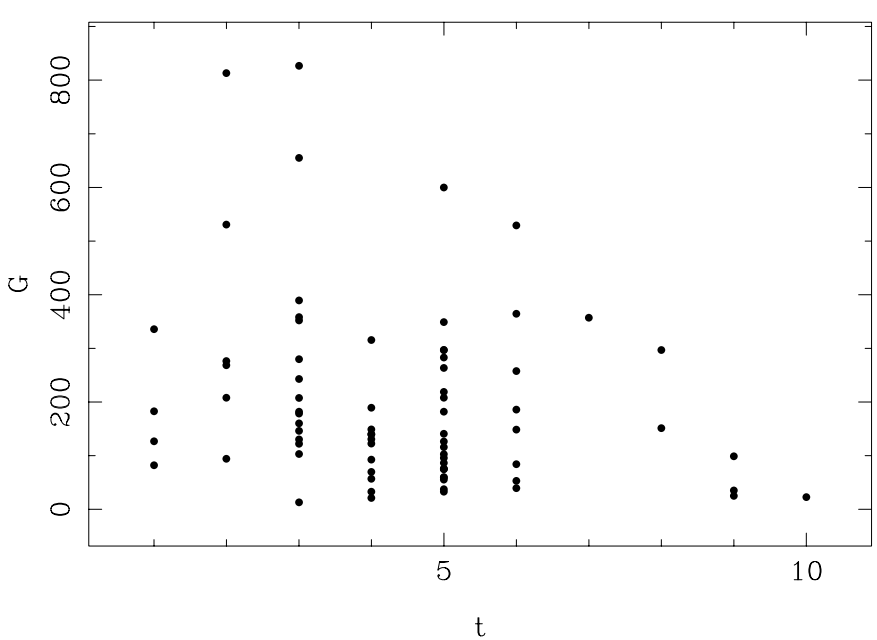

Fig. 8. Distribution of $G$ values (representing the inner solid-body rotation gradient) as a function of the morphological type, $t$.
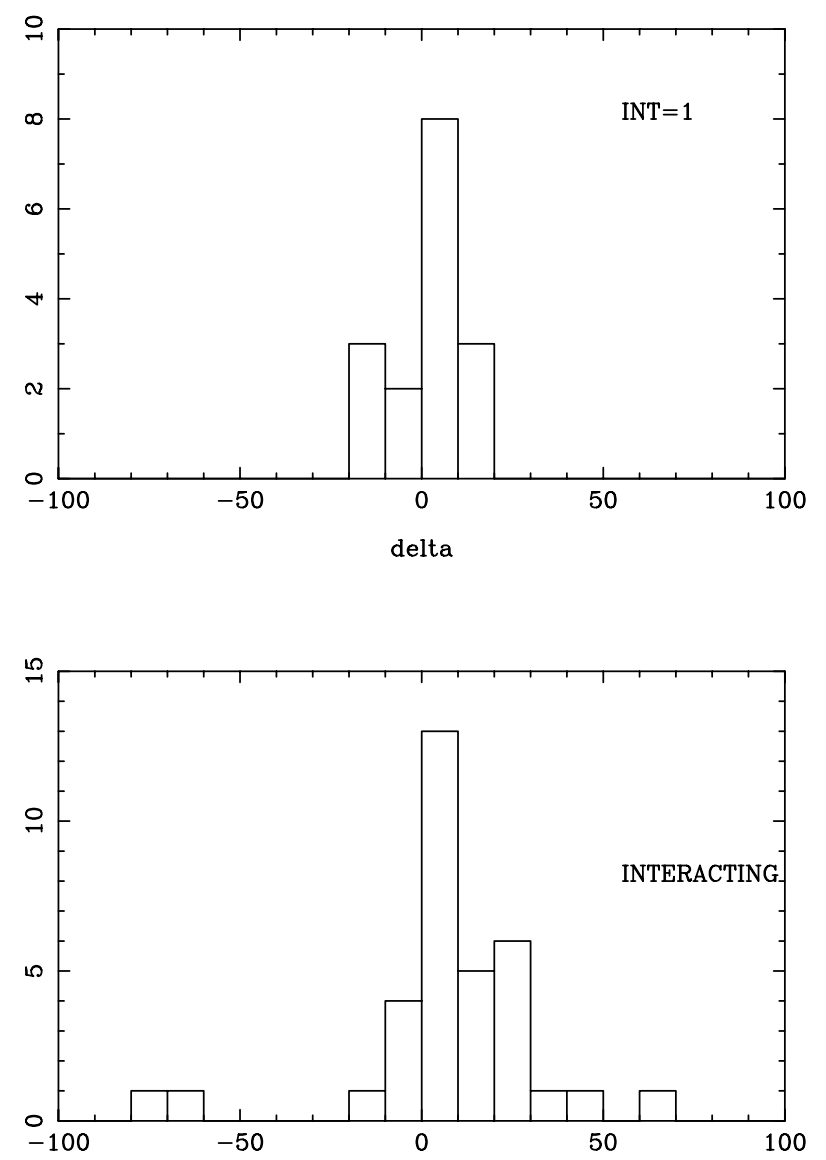

Fig. 9. Distribution of $\Delta$ values (representing the shape of the outer rotation curve.

(Rubin et al. 1991). We find the same tendency with the present data set (Fig. 10). The median values for type 1 to 6 are $223 \pm 46,253 \pm 29,194 \pm 41,160 \pm 33,186 \pm 37$, and $142 \pm 37$, respectively. The same $V_{\mathrm{m}}$ is related to the absolute magnitude (Fig. 11), with less scatter for the isolated objects.

We have further investigated whether there is the tendency found by Rubin et al. (1999) for later spiral galaxies to show more extended HII distributions, which translates

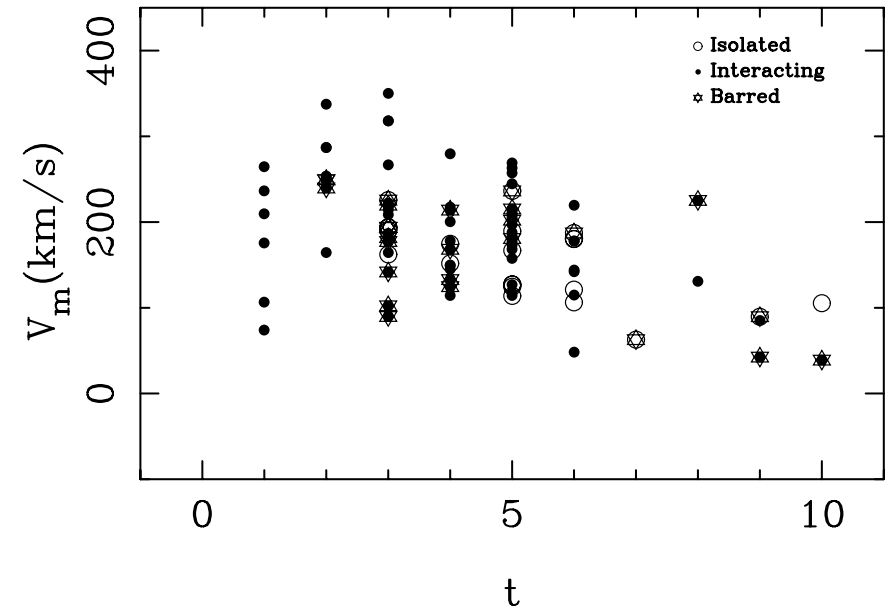

Fig. 10. Absolute maximum velocity versus morphological type, $t$.

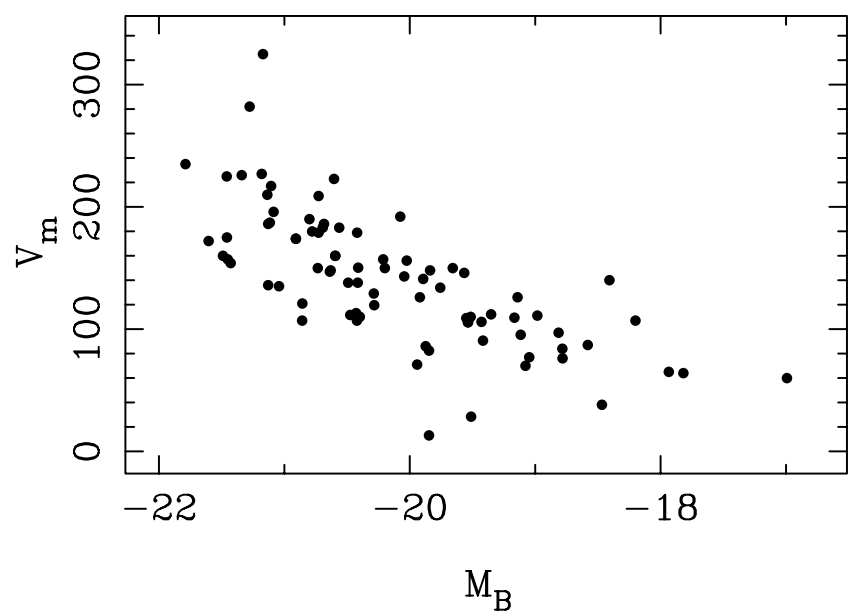

Fig. 11. Absolute maximum velocity as a function of absolute $B$ magnitude.

into longer rotation curves. This tendency is confirmed, as shown in Fig. 12, where the maximum extension of the rotation curve, $R_{\mathrm{M}}$ (in units of $R_{25}$ ) is plotted versus the morphological type. The increasing trend is clear for spirals up to $t=6$. No difference is found when the sample is separated according to the interaction state.

The central mass (inside the solid-body rotation region) seems to be slightly higher for early type galaxies, consistent with them hosting bigger bulges. No tendency is found between central mass and interaction class. The total masses within $R_{\mathrm{M}}$ and $R_{25}$ (this last from the RC3 catalogue), for an homogeneous and spherically symmetric distribution $\left(M_{\mathrm{T}}=\right.$ $2.3265 \times 10^{5} r v^{2}(r) M_{\odot}$, with $r$ in kpc and $v(r)$ in $\left.\mathrm{km} \mathrm{s}^{-1}\right)$ are also given. The $\mathrm{M} / \mathrm{L}$ relation is similar for galaxies with $t<7$, with no clear trend either with the morphological type, or with the interaction class.

\section{The Tully-Fisher relation}

As shown by Courteau (1997), the best kinematical tracer is $V_{2.2}$, which is the velocity attained at $R_{2.2} \approx 2.2 r_{\mathrm{d}}\left(r_{\mathrm{d}}=\right.$ disk scale length). Instead of $r_{\mathrm{d}}$ we have used $1.3 \times R_{\mathrm{eff}}$, equivalent 


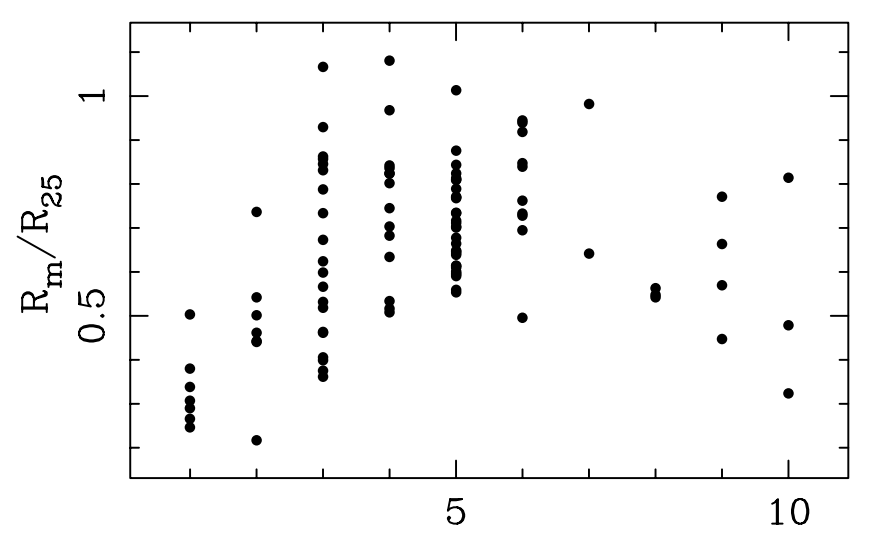

$\mathrm{t}$

Fig. 12. Maximum extension of the rotation curve, $R_{\mathrm{M}}$ (in units of $R_{25}$ versus the morphological type $t$.

to $0.65 \times R_{25}$, that results to be a good approximation for spiral galaxies and does not require a bulge-disk decomposition (Courteau 1997). Since the rotation curves derived from optical emission lines do not usually reach $R_{2.2}$, we fitted a model curve to the observed rotation curve in the inner part of the galaxy, and extrapolate it to the outermost regions. We have followed that procedure using models as simple as possible. We have used the normalized arctangent rotation curve fitting function given by

$v(R)=v_{0}+2 / \Pi \times v_{\mathrm{c}} \times \arctan (R)$

with $R=\left(r-r_{0}\right) / r_{\mathrm{t}}, v_{0}$ is the velocity center of rotation, $r_{0}$ is the spatial center of the galaxy, $v_{\mathrm{c}}$ is an asymptotic velocity and $r_{\mathrm{t}}$ is a radius that corresponds to the transition region between the rising and the flat parts of the rotation curve. This function has been shown to reproduce adequately the shape of the rotation curves with the smallest number of arguments (Courteau 1997) and emerges naturally from the standard parameterization of the density profiles of dark halos (Gilmore et al. 1990). As noted by Courteau (1997) the resulting parameters cannot be used to describe rotation amplitudes and scales, but since it allows to trace smoother curves and to somewhat extrapolate to $0.65 \times R_{25}$, it will be useful for our purposes. The model curves are shown in Figs. 4 and 5. According to this model, we have calculated the residuals, what allows us to obtain a quantitative estimation of the the goodness of the model: the rotation curves are classed as regular when the differences between the observed and the arctangent velocity distributions are smaller then $10 \%$; if these differences are higher than $10 \%$ but the shape of the curve still follows that of the model, the curve is classified as distorted; the rest are considered as peculiar. The curves may be also symmetric or asymmetric in the extension of the two branches. Therefore, the final classification is as following: 1: regular and symmetric; 2: regular and asymmetric; 3: distorted symmetric; 4: distorted asymmetric; 5 : peculiar.

The resulting TF relation is shown in Fig. 13, where the different interaction classes have been marked with different symbols. It can be seen that isolated galaxies trace the TF relation with the smallest scatter. Moreover, the three outliers belong to the group of interacting pairs. We have quantified the residu-

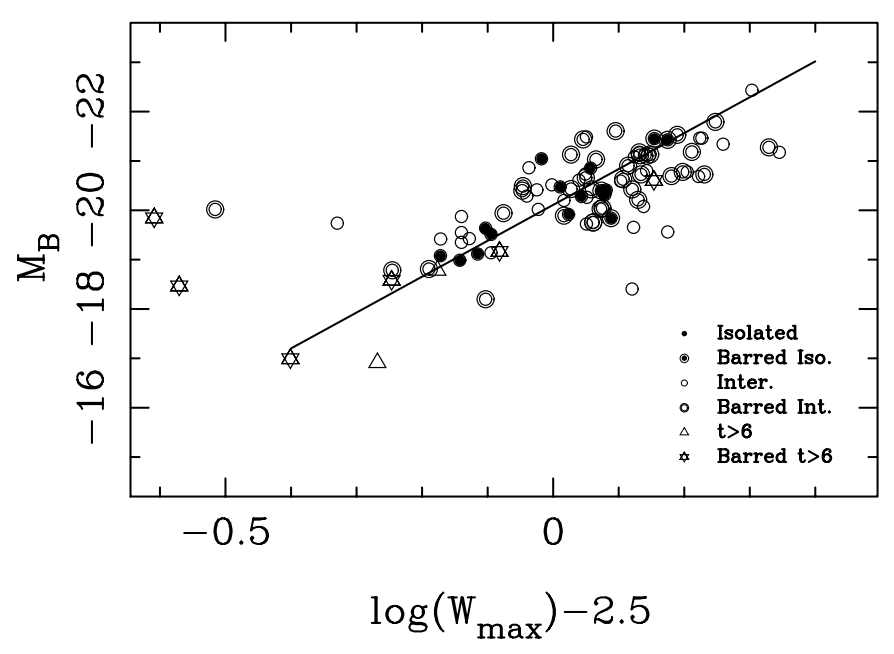

Fig. 13. TF relation for our sample galaxies. The solid line represents the TF line by Tully \& Pierce (2001).

als with respect to the TF relation as given by Tully \& Pierce (2000). We find 0.32 for isolated galaxies $(I N T=1)$ and 0.65 for interacting spirals $(I N T=3)$. We notice that the three outliers (NGC 2799, NGC 3395 and NGC 3396) show peculiar rotation curves (only NGC 2799 is not later than $t=6$, but is a secondary member), and that their position is much closer to the TF line when HI velocity amplitudes are considered. The same analysis has been applied to isolated and interacting objects. Therefore, even if the results shown here are only indicative due to small number statistics, we point out that a possibility seems to exist for reducing the scatter of the TF relation determined with optical data when using the most isolated objects.

We have also explored the eventual dependence of the departures from the TF line as a function of the degree of peculiarity of the rotation curves ${ }^{4}$. The dispersion from the canonic TF line is 0.30 for galaxies with regular rotation curves, 0.34 for galaxies with distorted rotation curves and 0.36 for peculiar rotation curves. This appears to be at variance with the results reported by Barton et al. (2001) who found that the presence of strong kinematic distortions is a significant predictor of TF residuals. The difference could be due to the fact that, as already pointed out, our sample includes only isolated and mildly interacting objects, that do not present strong distortions. Our data are in agreement with Dale et al. (2001) who find no differences between cluster and field spirals in the degree of asymmetry.

\section{Nuclear and disk spectral characteristics}

The distribution of the $\mathrm{H} \alpha$ line emission along the slit was traced for each 2D spectrum. The local peaks in the distribution were taken as the centers of HII regions, that were defined to comprise all the spatial sections within the FWHM around that peak. The resulting 1D spectra were measured as

\footnotetext{
${ }^{4}$ Notice that systematic effects in the third parameter analysis cannot not be addressed mainly due to the lack of accurate enough photometric information in our sample galaxies (see Kannappan et al. 2002).
} 
explained before, producing the data collected in Table 5. In the following the region comprising the center of the galaxy is referred as the Nuclear Region. Typical sizes for the Nuclear Regions range between 0.5 and $2 \mathrm{kpc}$. The spectra are not fluxcalibrated. However, since the lines used in the analysis are very close in wavelength, the count ratios are a good measure of the flux ratios.

Given the spectral coverage of the data, the standard diagnostic tools to classify the spectra (Baldwin et al. 1981; Veilleux \& Osterbrock 1987) cannot be used. But, as shown by the early work by Keel (1984), the [NII]/H $\alpha$ line ratio can be used as a rough, first order estimator to classify the spectral nuclear types $(S N T)$ into Active Galactic Nuclei (regardless of the kind of activity, since into the AGN category we have included both Seyfert and LINER-like nuclei) and HII like objects. Accumulated evidence has shown that this line ratio is very sensitive to the presence of any kind of activity, thus allowing for an easy spectral classification of the nuclei. The existence of absorption under the Balmer line could however induce the misclassification of some objects, and special care has to be taken.

Apart from a spectroscopic classification of the nuclear spectra, we have also attempted to give an estimation of the metallicity of the disk and its possible gradient, taking the $[\mathrm{NII}] / \mathrm{H} \alpha$ line ratio as an estimator. For low metallicity objects both nitrogen and oxygen are of primary origin and their abundances correlate well (Masegosa et al. 1994). For higher metallicities, a fraction of the measured Nitrogen is of secondary origin, which modifies the previous relation, even if it is still monotonic and, therefore, useful to probe $Z$. van Zee et al. (1998) have found that, for $12+\log (\mathrm{O} / \mathrm{H})<9.1$, a relation does exist of the form $12+\log (\mathrm{O} / \mathrm{H})=1.02 \log ([\mathrm{NII}] / \mathrm{H} \alpha)+9.36$. The use of the $[\mathrm{NII}] / \mathrm{H} \alpha$ line ratio to estimate $Z$ has the advantage of being insensitive to reddening. But, as Stasinska \& Sodré (2001) have pointed out, that calibration relation is adequate only for HII regions, and important errors could be produced when the integrated spectra of spiral galaxies, or a complex ISM with shocked gas is being analyzed. Based on the $[\mathrm{NII}] / \mathrm{H} \alpha$ ratio Denicoló et al. (2002) obtained an improved calibration of the oxygen abundances. They clearly showed the power of this estimator when analyzing large survey data to rank their metallicities, even if the uncertainties on individual objects can reach up to 0.6 dex, mainly due to $\mathrm{O} / \mathrm{N}$ abundance ratio and ionization degree variations. Here we only consider it to study global trends of $Z$ from the spectra of HII regions or HII-like nuclei in the collected sample of spiral galaxies.

\subsection{Nuclear spectra}

Nuclear $\mathrm{H} \alpha$ emission has been detected in 91 out of 98 galaxies in the sample. For the other 13, the stored data were corrupted. The nuclear spectra are presented in Fig. 21. Three of the galaxies without $\mathrm{H} \alpha$ emission (namely, NGC 3976, NGC 5641, and NGC 2424) show [NII] emission, suggesting that shock ionization would be important in these nuclei. They were classified as LINERs in previous studies (Carrillo et al. 1999), and they belong to the class of interacting systems. For the remaining

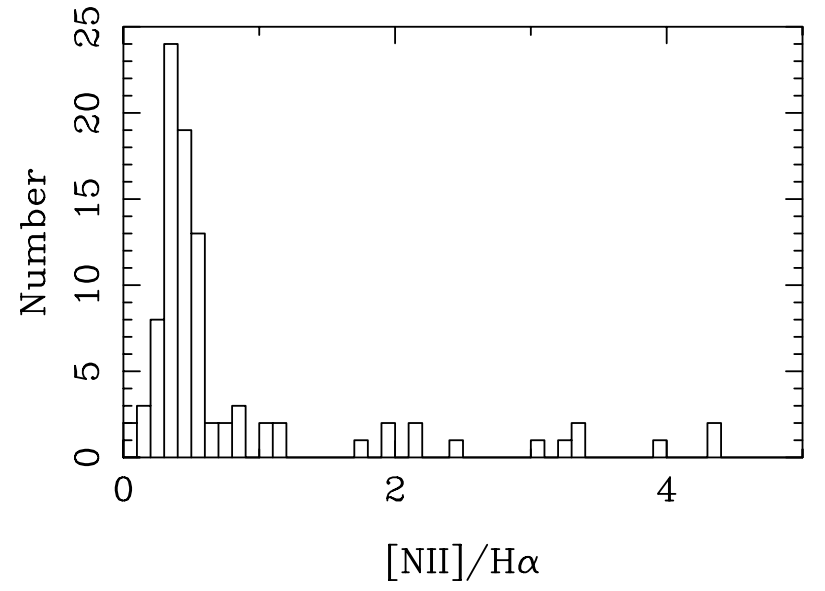

Fig. 14. Distribution of nuclear $[\mathrm{NII}] / \mathrm{H} \alpha$ for the whole sample. NGC 7217 has not been included due to the large measured ratio (see text).

4 galaxies (NGC 2344, NGC 2545, NGC 3835 and NGC 5147) only very faint or even absent emission has been detected partly due to the poor $S / N$ ratio of the spectra. In any case the emission cannot be strong. They do not show any other peculiarities and can be considered as normal spiral galaxies (Jansen et al. 2000).

Given the purpose of the work and the rather low $S / N$ ratio in many of the spectra we have not applied any correction for absorption. To cope with the problem of the presence of absorption under $\mathrm{H} \alpha$, we visually inspected all the spectra, identifying the cases where it was conspicuous. All those galaxies were classified as $S N T=3$, i.e., nuclei in which the Balmer absorption is so strong that the measured $[\mathrm{NII}] / \mathrm{H} \alpha$ ratio is not reliable to classify it. For the remaining nuclei, without any appreciable absorption under $\mathrm{H} \alpha$, those with spectral characteristics of HII regions were classified as $S N T=1$, and those with line ratios similar to active galaxies as $S N T=2$. Indeed this is a rather crude classification but, as we will see later, some conclusions on the nuclei of spiral galaxies and their relation to some global properties can be drawn.

The distribution of the $[\mathrm{NII}] / \mathrm{H} \alpha$ nuclear values shows that for most of the galaxies it is lower than 1 (see Fig. 14). The data are presented in Table 5. We notice that all the galaxies with $S N T=3$ have $E W(\mathrm{H} \alpha) \leq 10$, what produces an artificially high ratio if no correction is applied to cope with the underlying absorption and are consequently excluded hereafter from the discussion.

Judged from the $[\mathrm{NII}] / \mathrm{H} \alpha$ ratio, we find 11 AGN candidate objects (about 10\%) in our sample. Seven of them were already observed by Ho et al. (1997), who classified them as 6 LINERs and 1 Seyfert. For the remaining 4 galaxies we find that 2 of them are Seyfert 1 based on the width of the $\mathrm{H} \alpha$ line. For the other two the information we have is not enough to classify them as Seyfert 2 or LINER. The largest line ratio is found for N7217, with $[\mathrm{NII}] / \mathrm{H} \alpha=8.6$. It is a known LINER (Filippenko $\&$ Sargent 1985) frequently quoted to illustrate the signature of strong shocks (see the models by Dopita \& Sutherland 1995).

Regarding the HII-like nuclei, we find that they define a rather narrow distribution of the $[\mathrm{NII}] / \mathrm{H} \alpha$ ratio (Fig. 15). Only 


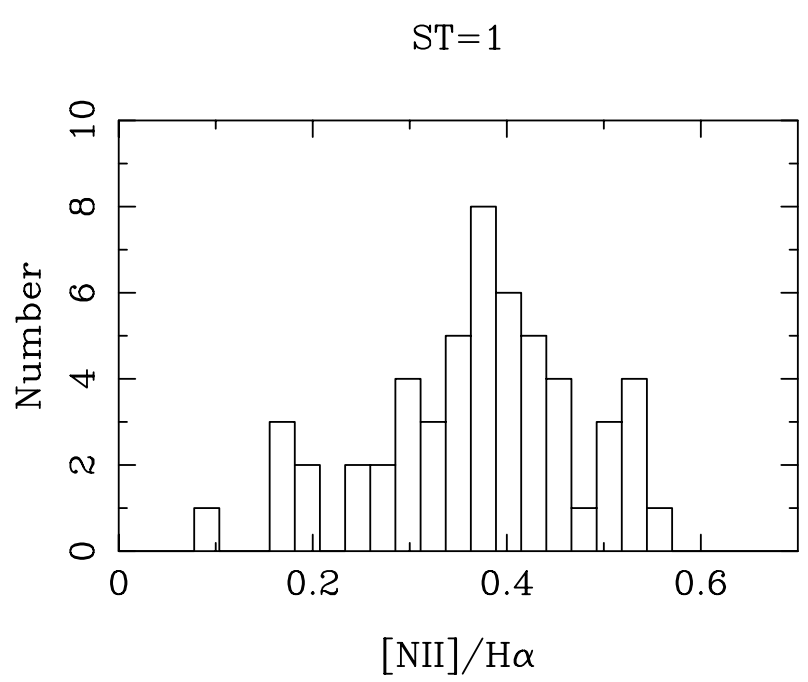

Fig. 15. Distribution of nuclear $[\mathrm{NII}] / \mathrm{H} \alpha$ for the galaxies with nuclear spectral type $S N T=1$.

two galaxies (not shown in the figure) depart from the general trend, N5172 with $[\mathrm{NII}] / \mathrm{H} \alpha=1.92$, and $\mathrm{N} 5678$ with $[\mathrm{NII}] / \mathrm{H} \alpha=1.16$. For N5172, our data are of very poor $S / N$ ratio and the $[\mathrm{NII}] / \mathrm{H} \alpha$ ratio we obtained is very uncertain. And N5678 is a composite LINER/HII galaxy after Filho et al. (2000). Excluding those two objects, the remaining galaxies present a range of values of the line ratio, corresponding to values typical of irregular galaxies and disk HII regions (see Vila-Costas \& Edmunds 1993 and McCall et al. 1985). For all the data classified as $S N T=1$, (with the quoted exceptions), included the latest spirals $(t>6)$, the median value is 0.38 , with a dispersion of 0.07. Excluding late type spirals it amounts to 0.39 with the same dispersion.

In spite of the rough character of the estimator we use here, some correlations are already hinted. There is a relation between the metallicity of the HII-like nuclei and the morphological type, the early type spirals having more metallic nuclei than the later spirals (Fig. 16, and Table 6). This result is consistent with the suggestion by Oey \& Kennicutt (1993) that early type spirals are more metal rich than later types. That results rests on the difference between $\mathrm{Sa} / \mathrm{Sab}$ galaxies in one side, and the later types in the other, since we don't find any difference between $\mathrm{Sb} / \mathrm{Sbc}$ and $\mathrm{Sc} / \mathrm{Scd}$ objects. Indeed, the later than Scd types are still of lower $Z$ and look like a different family. The results reported here are in agreement with the work by Zaritsky et al. (1994) based on a completely different data set, in the sense that a tendency does exist for the metallicity to decrease when moving along the Hubble sequence. We have investigated if such a trend could be due to a systematically stronger $\mathrm{H} \alpha$ absorption in early types. The absence of any appreciable trend between the $\mathrm{H} \alpha E W$ and the morphological type $\mathrm{t}$ argues against that explanation, and leaves the relation as genuine.

Finally, the analysis of a possible $Z$ enhancement produced by the presence of instabilities like bars or by the interaction with nearby neighbors has produced negative results. The range and median value of $Z$ does not seem to be altered when those aspects are taken into account.

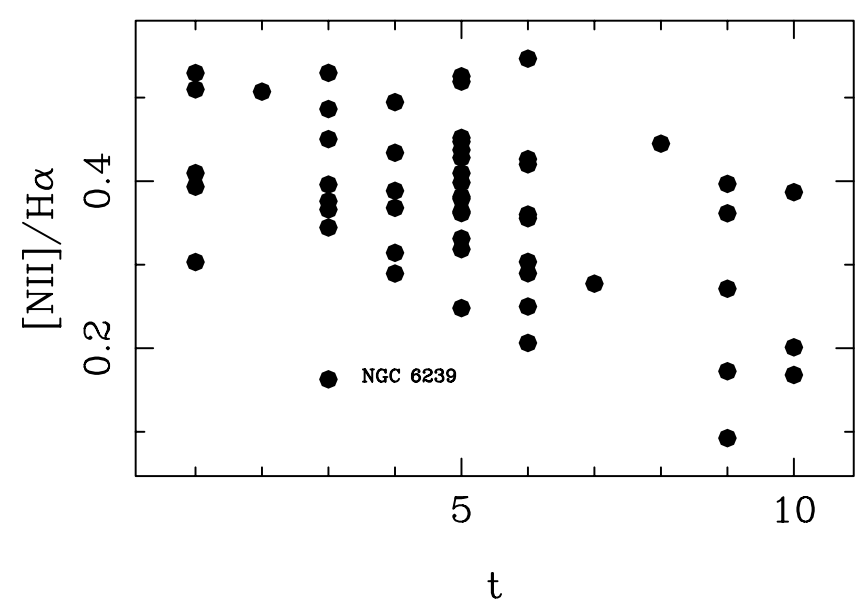

Fig. 16. $[\mathrm{NII}] / \mathrm{H} \alpha$ ratios as a function of the morphological type.

Table 6. $[\mathrm{NII}] / \mathrm{H} \alpha$ ratios for the three spectral nuclear types, and for the different morphological types.

\begin{tabular}{lc}
\hline \hline & {$[\mathrm{NII}] / \mathrm{H} \alpha$} \\
\hline$S N T=1$ & 0.38 \\
$S N T=2$ & 0.98 \\
$S N T=3$ & 0.76 \\
$t=1,2$ & 0.46 \\
$t=3,4$ & 0.38 \\
$t=5,6$ & 0.38 \\
$t>6$ & 0.27 \\
\hline
\end{tabular}

We have shown in Paper II that the relations are better defined if the type is replaced by a more quantitative parameter such as the $B / D$ ratio, or the inner gradient $G$, with which it is tightly correlated. Here too, we find a good correlation between $Z$ and the gradient $G$ (see Fig. 17), statistically significant at $99.99 \%$ confidence level ( $R=0.63$, for 38 objects). This adds to the previous finding about the quality of the parameter $G$ to characterize the global properties of spiral galaxies.

The other two global properties that appear to be related to the metallicity are the absolute magnitude, $M_{B}$ and the maximum rotation velocity. We find that $Z$ increases with both, the central velocity and the luminosity, i.e, massive galaxies are more metal rich. This agrees with the results reported by Zaritsky et al. (1994) and by Dutil \& Roy (1999). Again no difference is found between isolated and interacting galaxies. These results would suggest that the instabilities produced by gravitational interaction, even if they can drive gas to the center (Barnes \& Hernquist 1991; Mihos \& Hernquist 1996), do not have major effects in the central region for mild interaction as the ones reported in this work.

\subsection{Extranuclear HII regions}

The extranuclear HII regions detected in all our 2D spectra were measured and used to characterize the metallicity of the disks. As discussed before, our data does not allow us to conclude on any individual galaxy, but can be used to look for general trends when the population of the disks is considered as 


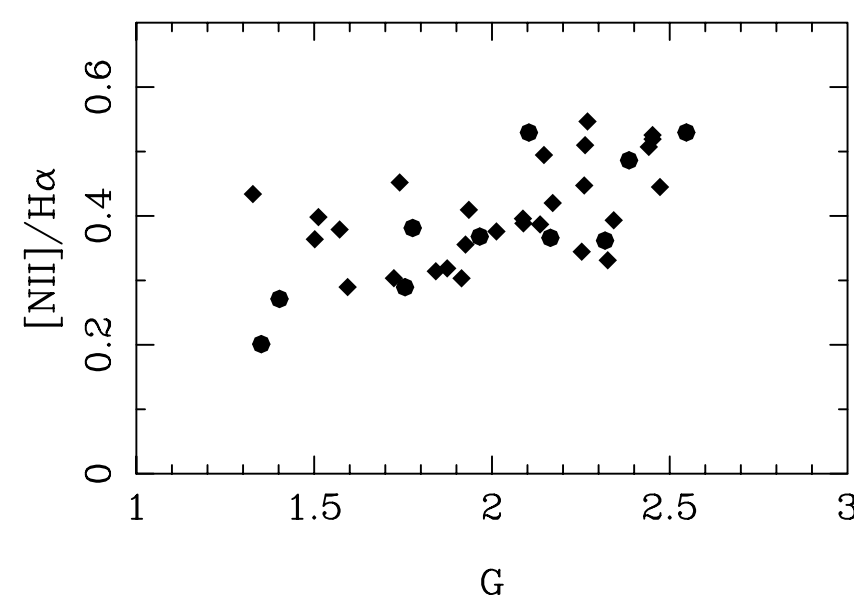

Fig. 17. Nuclear $[\mathrm{NII}] / \mathrm{H} \alpha$ ratios as a function of the gradient of the solid-body region of the rotation curve, $G$ ( $G$ is in logarithmic scale). Interacting galaxies are marked with solid circles.

a whole. To be able to combine data from different galaxies, we have normalized to $R_{25}$ (as given in the RC3) the galactocentric distances of the HII regions. In principle the choice of one or another radius to normalize could have some effect on the results of $Z$ gradients (Zaritsky et al. 1994). Since we are interested in general trends and not in a real quantification of the gradient we consider that the choice of the normalization is of minor importance and decided to use the isophotal radius, accessible for most of the galaxies studied. From the HII regions measured we have only selected all the data with $\mathrm{H} \alpha$ equivalent width larger than $10 \AA$. In that way we select the better $S / N$ data, and avoid including regions with important Balmer absorption, that could induce inconsistencies in the estimation of the metallicity. The number of regions we consider here is 392, in 98 galaxies. In the following we report the results obtained when the general metallicity trends in the disk are analyzed in relation to the morphology of the galaxy, the Nuclear Spectral Type and the effects produced by interaction. Different authors have claimed (see Vila Costas \& Edmunds 1992 for a review and Zaritsky et al. 1994) that a $Z$ radial gradient does exist in disk like galaxies. In Fig. 18 the metallicity estimator $[\mathrm{NII}] / \mathrm{H} \alpha$ is presented for the different morphological types. A slight tendency seems to be present for the gradient to be steeper in later types, whereas it is about zero for $\mathrm{Sa}$ and $\mathrm{Sb}$ spirals. This agrees with the claim by Oey \& Kennicutt (1993) of a larger global metallicity and almost flat gradients in early type spirals.

We have calculated from the disk data the expected central $[\mathrm{NII}] / \mathrm{H} \alpha$ values, using the formal fitting to the data. The values we find in that way ranges from 0.41 for the earlier types, to 0.28 for the latest types. They compare very well with what we have found just measuring the line ratio of the nuclear regions, 0.44 for the early types, and 0.27 for the latest types. That consistency adds confidence to the reality of the trends we have found, and to the way of estimating the metallicity form the line ratio.

The $\mathrm{H} \alpha$ Balmer line has been extensively used to measure the ratio of the current to the average past Star Formation Rate in Galaxies (see Kennicutt 1983; Kennicutt et al. 1994;
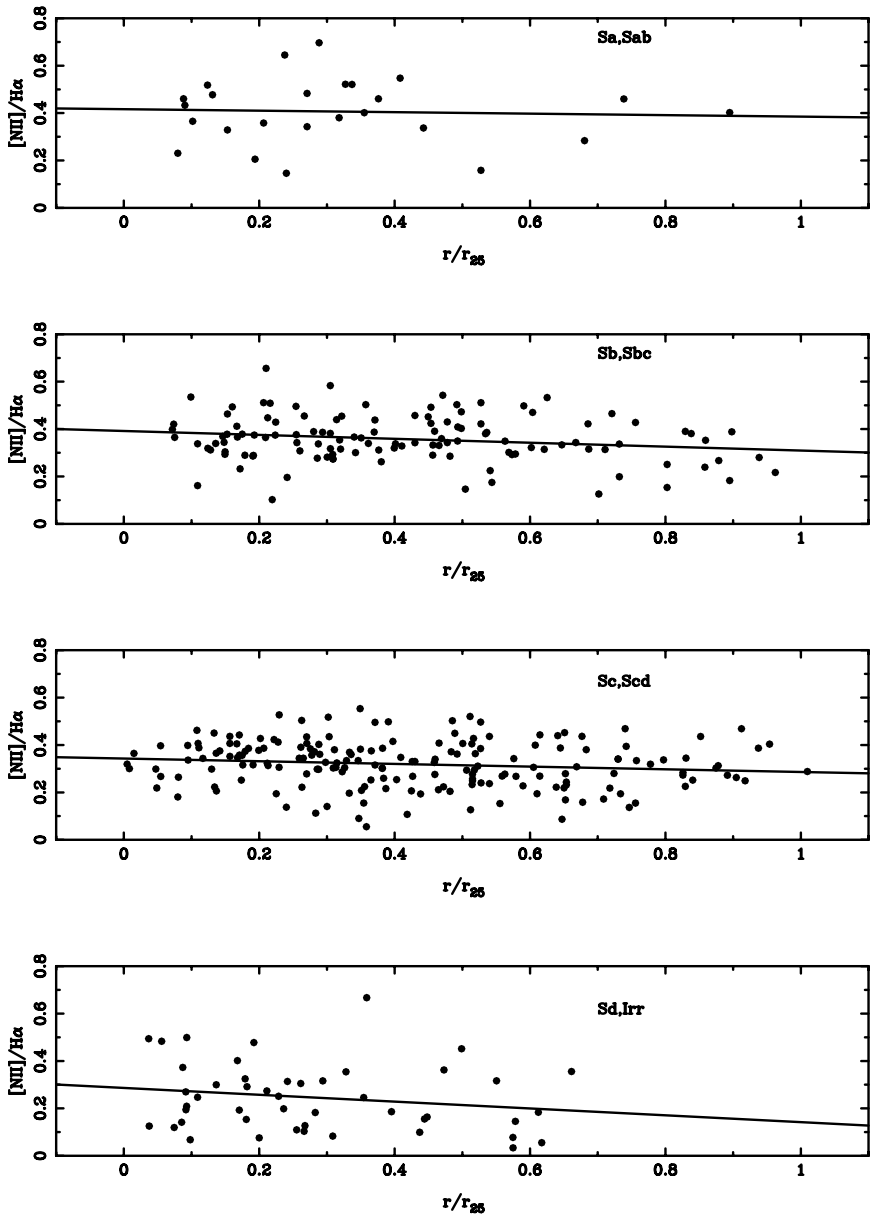

Fig. 18. Disk $[\mathrm{NII}] / \mathrm{H} \alpha$ ratios as a function of the distance to the center (normalized to $R_{25}$ ).

Stasinska \& Sodré 2001). Kennicutt (1994) found smooth progression in the Star Formation History with the Hubble type, with a ratio of current to past SFR increasing from $0.01-0.1$ for Sa type to $0.5-2$ for a typical Sc disk. The data reported here are only barely consistent with such a claim. In Fig. 19, where the $\mathrm{H} \alpha$ equivalent width is plotted versus the radial distance, the only effect is a larger dispersion on later types than in earlier spirals towards larger $E W$ in the later types but a clear separation between different morphologies is not obvious. It has to be noticed that Kennicutt data refer to the integrated $E W$ whereas here we are trying to get the trend based on the distribution of HII regions crossed by the slit through the disk of the galaxies. We cannot extract a definitive conclusion from our data and, therefore, we cannot say that our data are in contradiction with Kennicutt's study, even if such a conclusion is hinted at by our results.

Let's now compare isolated and interacting systems. Regarding the metallicity, it appears that interacting galaxies tend to show a larger $[\mathrm{NII}] / \mathrm{H} \alpha$ ratio in all the mapped regions (see Fig. 20). The median value of $[\mathrm{NII}] / \mathrm{H} \alpha$ for the disk of isolated normal galaxies is 0.27 , in contrast with a median value of 0.35 for the interacting systems.

It is usually accepted that the interaction process drives gas to the central regions producing an enhancement in the star 


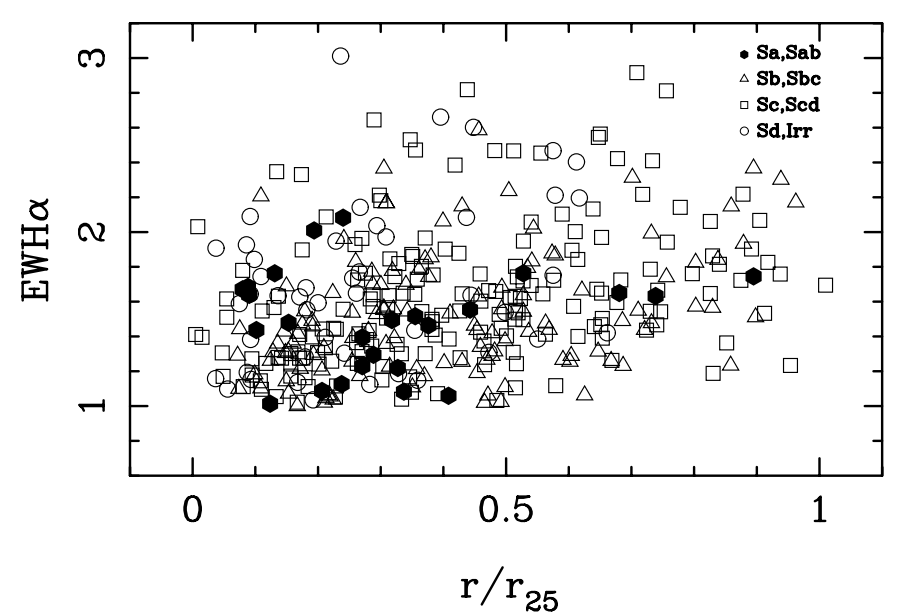

Fig. 19. Disk equivalent widths of $\mathrm{H} \alpha$ (in $\AA$ ) as a function of the morphological type, $t$. EWs are plotted in logarithmic scale.

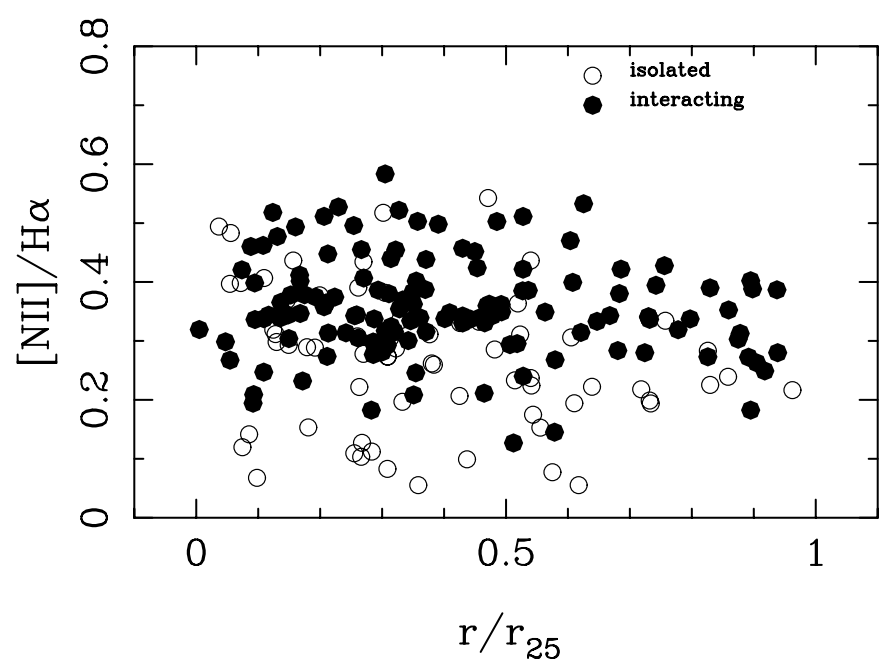

Fig. 20. Metallicity estimator for the HII regions in the disks of isolated and interacting spirals.

formation events. Consequently, a larger $Z$ in the bulges of those galaxies would be predicted, and it's found in our data and other studies. The point here is that we have also found a higher $Z$ along the disks of the same galaxies. It seems then that the interaction affects the whole galaxy, producing star formation in all the disk, depending on the conditions (see for example, Márquez \& Moles 1994).

However, no difference in the $\mathrm{H} \alpha E W$ has been found between interacting and isolated galaxies, as if the global star formation rate now was essentially the same in both families. To understand this result we have to take into account that only mildly interacting systems are included in our sample, for which the effects of the interaction are expected to be much less important than in stronger interactions. In that sense, we notice that our results are compatible with those found by Kennicutt et al. (1987) for a large fraction of galaxies in their complete pairs sample. Combining both results, higher $Z$ and normal present star formation rate, it seems that the enrichment is only produced as a secular, accumulative effect along the galaxy life, without marked episodes, in those mildly interacting systems.
This result is consistent with those by Bergvall et al. (2001), who find reddest disks in interacting galaxies. Nevertheless, we have already noticed that the morphological types of interacting galaxies tend to be earlier than for isolated ones, so the reported higher metallicities could be reflecting the difference in metallicities between early and late type galaxies. Larger samples of isolated galaxies would be needed to further analyze the metallicities of early types spirals as compared to those of interacting spirals with the same morphologies.

The situation for AGNs is somewhat similar, since active spirals are known to mainly reside in early type spirals (see for instance Moles et al. 1995). Given the limited sample we are considering (11 AGNs, 7 of them belonging to interacting systems) eventual differences in metallicity cannot be addressed.

\section{Summary and conclusions}

We have obtained long slit spectra along the major axes of a sample of spiral galaxies selected to be either isolated or in isolated pairs, with similar intermediate-scale environment and with a recognizable and well defined spiral morphology. We have further investigated their environmental status and reclassified them, what allows us to define a sample of isolated objects, to be later compared with mildly interacting spirals (with small satellites and/or companions of similar size). The main results we have obtained are the following:

- We have confirmed previous results (Paper II) that isolated galaxies tend to be of later Hubble types and lower luminosity than the interacting galaxies.

- the outer parts of the rotation curves of isolated galaxies tend to be flatter than in interacting galaxies. They show similar relations between global parameters. The scatter of the Tully-Fisher relation defined by isolated galaxies appears to be significantly lower than that of interacting galaxies.

- there is a clear trend between the metallicity of the HII-like nuclei and the morphological type of the galaxy, the earlier types showing larger $Z$ values. Extrapolation of the $Z$-trend in the disk to the central position gives consistent results with the direct measurement of the nuclear HII region. No trend with the interaction status was found.

- we report here for the first time the existence of a tight correlation between $Z$ and the gradient of the inner rigid solid rotation part of the rotation curve, $G$.

- the $Z$-gradient of the disks depends on the type, being almost flat for early spirals, and increasing for later types.

- [NII]/H $\alpha$ ratios appear to be larger for disk HII regions interacting galaxies. This could be simply due to the fact that early types are more frequent among them. (A similar result is obtained for AGNs, but we cannot further test it given the small size of our sample.) On the other hand, the $\mathrm{H} \alpha E W$ present similar values in all kind of galaxies. At face value those results would indicate that mildly interacting galaxies (such as those in our sample) have different histories form normal galaxies. This difference however has no marked 
episodes (bursts) of star formation, but only small cumulative effects that result in more metallic (and redder) disks and nuclei.

Acknowledgements. We are very grateful to the anonymous referee, whose comments and suggestions helped us improve the presentation. We thank Prof. G. Paturel who kindly made available to us prior to publication the Galaxy Catalog we have used to determine the interaction status of our sample galaxies. We also thank L. Cariggi for her careful reading of the manuscript and valuable comments. I. Márquez acknowledges financial support from the Spanish Ministerio de Ciencia y Tecnología and the IAA. D. Jesús Varela acknowledges a scholarship from the Ministerio de Ciencia y Tecnología. This work is financed by DGICyT grants PB93-0139, PB96-0921, PB98-0521, PB98-0684, ESP98-1351, AYA2001-2089 and the Junta de Andalucía. This research has made use of the NASA/IPAC extragalactic database (NED), which is operated by the Jet Propulsion Laboratory under contract with the National Aeronautics and Space Administration.

\section{References}

Athanassoula, E. 1984, Phys. Rep., 114, 319

Amram, P., Balkowski, C., Boulesteix, J., et al. 1996, A\&A, 310, 737 Baiesi-Pillastrini, G. C. 1987, A\&A, 1972, 375

Baldwin, J. A., Phillips, M. M., \& Terlevich, R. 1981, PASP, 93, 5

Barnes, J. E., \& Hernquist, L. E. 1991, ApJ, 370, 65

Barton, E., Geller, M. J., \& Kenyon, S. J. 2000, ApJ, 530, 660

Barton, E., Geller, M. J., Bromley, B. C., et al. 2001, AJ, 121, 625

Bergvall, N., Laurikainen, E., \& Aalto, S. 2001, A\&A, submitted

Byrd, G. G., \& Howard, S. 1992, AJ, 103, 1089

Carrillo, R., Masegosa, J., Dultzin-Hacyan, D., \& Ordoñez, R. 1999, Rev. Mex. A\&A, 35, 187

Conselice, C. J., \& Gallagher, J. S., III 1999, AJ, 117, 75

Conselice, C. J., Bershady, M. A., \& Jangren, A. 2000, ApJ, 529, 886

Courteau, S. 1997, AJ, 114, 2402

Dale, D. A., Giovanelli, R., Haynes, M., et al. 2001, AJ, 121, 1886

Denicoló, G., Terlevich, R., \& Terlevich, E. 2002, MNRAS, 330, 69

Dopita, M. A., \& Sutherland, R. S. 1995, ApJ, 455, 468

Dutil, Y., \& Roy, J.-R. 1999, ApJ, 516, 62

Filho, M. E., Barthel, P. D., \& Ho, L. C. 2000, ApJS, 129, 93

Filippenko, A. V., \& Sargent, W. L. W. 1985, ApJS, 57, 503

Ferguson, A. M. N., Gallagher, J. S., \& Wyse, R. F. G. 1998, AJ, 116, 673

Gilmore, G. F., King, I. R., \& van der Kruit, P. C. 1990, The Milky Way as a Galaxy, ed. R. Buser, \& I. R. King (University Science Books, CA), 212
Henry, R. B. C., \& Worthey, G. 1999, PASP, 111, 919

Ho, L. C., Filippenko, A. V., \& Sargent, W. L. W. 1997, ApJS, 112, 315

Huchra, J. P., Vogeley, M. S., \& Geller, M. J. 1999, The CfA2s catalog, VizieR On-line Data Catalog: J/ApJS/121/287

Jansen, R. A., Fabricant, D., Franx, M., \& Caldwell, N. 2000, ApJS, 126,331

de Jong, R. S., \& van der Kruit, P. C. 1994, A\&AS, 106, 451

Kannappan, S. J., Fabricant, D. G., \& Franx, M. 2002, AJ, 123, 2358

Karachentsev, I. D. 1972, Soob. Sb. Astr. Observatory Akad, Nauk, 7

Keel, W. C. 1984, ApJ, 282, 75

Keel, W. C. 1993, AJ, 196, 1771

Keel, W. C. 1996, ApJS, 106, 27

Kennicutt, R. C., Jr., et al. 1987, AJ, 93, 1011

Kennicutt, R. C., Jr. 1983, ApJ, 272, 54

Kennicutt, R. C., Jr., Tamblyn, P., \& Congdon, C. E. 1994, ApJ, 435, 22

Kraan-Korteweg, R. C. 1986, A\&AS, 66, 255

Márquez, I. 1994, Ph.D. Thesis, Universidad de Granada

Márquez, I., \& Moles, M. 1994, AJ, 108, 90

Márquez, I., \& Moles, M. 1996, A\&AS, 120, 1 (Paper I)

Márquez, I., \& Moles, M. 1999, A\&A, 344, 421 (Paper II)

Masegosa, J., Moles, M., \& Campos-Aguilar, A. 1994, ApJ, 420, 576

Mathewson, D. S., Ford, V. L., \& Buchorn, M. 1992, ApJS, 81, 413

McCall, M. L., Rybski, P. M., \& Shields, G. A. 1985, ApJS, 57, 1

Moles, M., Márquez, I., \& Pérez, E. 1995, ApJ, 438, 604

Mihos, J. C., \& Hernquist, L. 1996, ApJ, 464, 641

Oey, M. S., \& Kennicutt, R. C., Jr. 1993, ApJ, 411, 137

Persic, M., Salucci, P., \& Stel, F. 1996, MNRAS, 281, 27

Peterson, Ch. J., Rubin, V. C., Ford, W. K., \& Roberts, M. S. 1978, ApJ, 226, 770

Rubin, V. C., Hunter, D., \& Ford, W. K. 1991, ApJS, 76, 153

Rubin, V. C., Kenney, J. D. P., \& Young, J. S. 1997, AJ, 113, 1250

Rubin, V. C., Waterman, A. H., \& Kenney, J. D. P. 1999, AJ, 118, 236

Stasinska, G., \& Sodré, L. 2001, A\&A, 374, 919

Sofue, Y. 1998, ApSS, 269, 593

Sundelius, B., Thomasson, M., Valtonen, M. J., \& Byrd, G. G. 1987, A\&A, 174, 67

Tully, R. B., \& Pierce, M. J. 2000, ApJ, 533, 744

van Zee, L., Salzer, J. J., Haynes, M. P., O’Donoghe, A. A., \& Balonek, T. J. 1998, AJ, 116, 2805

Veilleux, S., \& Osterbrock, D. E. 1987, ApJS, 63, 295

Vila-Costas, M. B., \& Edmunds, M. G. 1993, MNRAS, 265, 199

Zaritsky, D., Kennicutt, R. C., Jr., \& Huchra, J. P. 1994, ApJ, 420, 87 